\title{
Chebyshev Polynomial-Based Authentication Scheme in Multiserver Environment
}

\author{
Toan-Thinh Truong $\mathbb{C}^{1,2}$ Minh-Triet Tran, ${ }^{1,2}$ and Anh-Duc Duong ${ }^{2,3}$ \\ ${ }^{1}$ Information Technology, University of Science, Ho Chi Minh City 700000, Vietnam \\ ${ }^{2}$ Vietnam National University, Ho Chi Minh City 700000, Vietnam \\ ${ }^{3}$ University of Information Technology, Ho Chi Minh City 700000, Vietnam \\ Correspondence should be addressed to Toan-Thinh Truong; ttthinh@fit.hcmus.edu.vn
}

Received 11 November 2019; Accepted 6 May 2020; Published 25 August 2020

Academic Editor: Jiankun $\mathrm{Hu}$

Copyright (C) 2020 Toan-Thinh Truong et al. This is an open access article distributed under the Creative Commons Attribution License, which permits unrestricted use, distribution, and reproduction in any medium, provided the original work is properly cited.

\begin{abstract}
Nowadays, communication technologies are more and more strongly advanced, such as 4G or 5G. There are many useful online applications, and one of them is the telecare medical information system (TMIS). If the TMIS is widely deployed, patients and doctors will have more frequently connection. Clearly, this enhances our quality of life. One of the most important modules securely constructing this convenient TMIS is the user-authentication scheme. We should prevent user identity and related information from the adversary's eavesdropping. Therefore, the authentication scheme could provide user anonymity and concern some kinds of attacks, such as impersonation or password-guessing attacks. Common solutions are a combination of hash function and public-key cryptosystem (RSA or elliptic curve cryptosystem, ECC), but current schemes do not consider identity protection as one main task necessary for medical information environment. In this paper, our results consist of some important analyses of previous works and a multiserver user-authentication scheme suitable for TMIS using Chebyshev polynomial with two models: random oracle and BAN-logic.
\end{abstract}

\section{Introduction}

With evolutionary changes in technological fields, all aspects of modern life are influenced positively, especially in medical online-service systems. Internet gives us a chance of providing convenience to our customers. Instead of directly coming to the medical centre or hospital, many people like to experience anytime. Nowadays, people use wearable devices, such as smart watch or bracelet, and make connections with the online medical system to quickly receive some doctors' advises. It can be said that remote services are an inevitable trend to satisfy remote experiences. In such services, we need to protect the users' profiles from illegitimate accesses. All exchanged messages between the user and server in a working session need keeping secret. In any application, the user and server must know if their partner is real or fake.
Therefore, the authentication scheme is necessary to provide security and privacy for both sides.

Storing a password list to verify the user's identity is a popular method, and this is not a secure one (PAP/CHAP). This list may be stolen, and then another adversary can launch a password dictionary attack. Furthermore, the information exchanged between the user and server must be kept secure. We need to propose an efficient scheme to overcome some existing limitations. To achieve this goal, we should design an authentication scheme combined with some cryptographic primitives and hard problems to resist some common kinds of attacks. However, many authors prefer the password-based approach to others because it is simple and easily deployed. Some schemes [1-5] can resist some kinds of attacks at this phase, such as stolen-verifier attack or replay attack. In $2010, \mathrm{Wu}$ et al. [6] proposed a 
scheme with precomputing phase enhancing the security. The remarkable point of this idea is that a set of prestored random values provides a strong user's anonymity. Furthermore, authors also use some cryptographic primitives, such as hash function, symmetric encryption scheme, and logarithm problem. Then, Debiao [7] pointed out that Wu's scheme did not combine the user's identity with secret information, and this results in impersonation attack. What Debiao claimed is true, but his improved scheme still has this pitfall. Next, Wei [8] discovered that both Debiao and $\mathrm{Wu}$ are vulnerable to offline password-guessing attack, and he also proposed improved version to overcome this attack. In 2012, Zhu claimed that Wei's scheme is still vulnerable to what Wei claimed. Zhu combined the password with a secret key to enhance the difficulty of password verification. Although Zhu's scheme [9] overcame previous limitations, his scheme transmitted identity information without protection. Therefore, his scheme is not suitable for some privacy environments. Especially, Pu's plugin scheme [10] can plug any two-party password authentication protocol, 2PAKE with elliptic curve cryptography, to enhance security and save computational cost. However, this scheme also needs to be reconsidered because of unreasonable computation workloads with two session keys. In case of leaking the centre's master key and the users' authentication key, the scheme should protect previous exchanged messages between the user and server. That is why session-key perfect forward secrecy (PFS) is one of the standards evaluating a strong scheme. Known-key attack is also a popular one at the authentication phase that receives many attentions. In this kind of attack, leaking another session key may result in attacking another session key. In 2013, Li et al. [11] proposed a scheme in multiserver environment with many improvements, in which each server has its own key. However, leaking smart card's information may result in passwordguessing attack. In 2014, Qu and Tan [12] proposed a different ECC-based scheme. Although they used elliptic curve cryptosystem, leaking the user's identity may result in impersonation attack. Clearly, this decreases the scheme's reality because the identity's nature is public. In 2015, Amin and Biswas [13] proposed a scheme in telecare medicine environment. Their scheme can resist three-factor attack, including password + smart card + biometrics. However, their scheme is still vulnerable to PFS. In 2018, Qiu et al. [14] and $\mathrm{Xu}$ et al. [15] proposed a scheme using ECC with untraceability property suitable for the medical services. Also, in 2019, Qiu et al. [16] proposed an ECC-based improved version using automated validation of Internet security protocol and application software. So, it can be said that this scheme has a high reliability.

Client-server authentication is simple and time-efficient, but in such medical or financial systems, we need continuous connections between their servers. Furthermore, in singleserver environment, the customer needs many credentials for various services. Recently, using Chebyshev polynomial receives attentions from many authors. In 2016, Li et al. [17] proposed a chaotic map-based authentication scheme in multiserver environment with provable security. Their work is truly impressive because it is based on BAN-logic and random oracle models, which are tools suitable for provable authentication schemes. Their design is a three-party participation in authentication process, so its time-consuming is high. In 2017, Jangirala et al. [18] proposed a multiserver environment scheme based on dynamic ID. Although the correctness of their scheme is correctly proved based BANlogic, it is not applied with any hard problems. Therefore, it is hard to be a strong scheme. In the same year, Han et al. [19] and Irshad et al. [20] proposed a chaotic map-based scheme. Han et al.'s result is a combination between hard problem (chaotic map) and cryptographic primitives, such as hash function and symmetric encryption scheme. However, we see their scheme uses three-way challengeresponse handshake technique with timestamp. In our experience, we only need two three-way challenge-response handshake techniques needed if using timestamp. Irshad's scheme is similar to Li's because it is designed with threeparty architecture. Therefore, it also takes much time to authenticate. In 2018, Alzahrani et al. [21] proposed a secure and efficient TMIS-based scheme. Their scheme provides TMIS environment with chaotic map-based scheme, but they need to extend in multiserver environment. Especially, in the same year, Wang et al. [22] proposed a security model accurately capturing the adversary's practical capabilities. We hope their model will be favourable and common soon. In this paper, we will analyse typical works [11-13, 18, 20, 21] to have some information needed to propose a new Chebyshev polynomial-based scheme in multiserver environment. Also, we have a work [23] but in the client-server environment.

The rest of our paper is organized as follows. In Section 2, we present the background of Chebyshev polynomial. Section 3 reviews some recently typical results and analyses them on security aspect. Then, in Section 4, we propose an improved scheme in multiserver environment using Chebyshev polynomial [24] in the modular prime number field. In Section 5, we analyse our proposed scheme on two aspects, security and efficiency. Finally, the conclusion is presented in Section 6.

\section{Background}

Chebyshev polynomial [24] is a chaotic map in field $\mathbb{R}, T_{a}$ : $[-1,1] \longrightarrow[-1,1](\forall a \in \mathbb{N}): T_{a}(x)=\cos (a \times \operatorname{arcos}(x)), \forall x \in$ $[-1,1]$.

And it can be rewritten in recursion form as follows:

$$
\left\{\begin{array}{l}
T_{1}(x)=x \\
T_{2}(x)=2 x^{2}-1, \\
T_{3}(x)=4 x^{3}-3 x \\
T_{4}(x)=8 x^{4}-8 x^{2}+1, \\
\quad \vdots \\
T_{a+1}(x)=2 x \times T_{a}(x)-T_{a-1}(x), \quad \forall a \in N .
\end{array}\right.
$$

In 2005, Bergamo et al. [25] analysed Chebyshev polynomial in real field and concluded that we can find $r^{\prime} \neq r$, such that $\operatorname{Tr}^{\prime}(x)={ }_{T r}(x)$. In 2008, Zhang [24] extended 
Chebyshev polynomial to $\infty$ and proved that its property in real field is also right in modular prime number field $\mathbb{Z}_{p}$, $p \in \mathbb{P}$. This result allows to construct public-key cryptography and related hard problems. Chebyshev polynomial in $\mathbb{Z}_{p}$ can be rewritten in recursion form as in $\mathbb{R}$ :

$$
\left\{\begin{aligned}
T_{0}(x) & =1, \\
T_{1}(x) & =x, \\
\vdots & \\
T_{n}(x) & =2 x \times T_{n-1}(x)-T_{n-2}(x) \bmod p, \quad \forall n \geq 2 .
\end{aligned}\right.
$$

With properties in Chebyshev polynomial, a public-key cryptography is proposed. To construct this one, we need to choose $p \in \mathbb{P}$ and $x \in[0, p-1]$ and then compute with formula $T_{n}(x) \bmod p, \forall n \in \mathbb{N}$. Furthermore, there are also two related hard problems in this public-key cryptography [26], such as chaotic map discrete logarithm problem (CMDLP) and chaotic map Diffie-Hellman problem (CMDHP):

(i) Chaotic map discrete logarithm problem (CMDLP): given $p \in \mathbb{P}$ and $x, y \in[0, p-1]$, it is hard to find $r \in \mathbb{N}$ such that $T_{r}(x)=y \bmod p$

(ii) Chaotic map Diffie-Hellman problem (CMDHP): given $p \in \mathbb{P}, x \in[0, p-1], T_{a}(x) \bmod p$ and $T_{b}(x)$ $\bmod p$, it is hard to find $T_{a \times b}(x) \bmod p$, where $a$, $b \in \mathbb{N}$

\section{Cryptanalysis of Some Typical Schemes}

This section presents and analyses on some typical schemes.

3.1. Li et al.'s Scheme. This scheme [11] uses hash function combined with random values, including four phases: registration, login, authentication, and password-update phases. Because designed for multiserver environment, the registration centre constructs the master key $h(x \| y)$ for itself and the submaster key $h\left(S I D_{j} \| h(y)\right)$ for each service provider. Table 1 presents some notations used in this scheme.

\subsubsection{Registration Phase. $U_{i}$ registers with $R C$ as follows:}

(i) $U_{i}$ chooses $I D_{i}, P W_{i}$, and random value $b$ and computes $A_{i}=h\left(b \oplus P W_{i}\right)$. Then, $U_{i}$ sends $I D_{i}$ and $A_{i}$ to $R C$ through a secure channel.

(ii) On receiving $\left\{I D_{i}, A_{i}\right\}$ from $U_{i}, R C$ computes $B_{i}=h$ $\left(I D_{i} \| x\right), C_{i}=h\left(I D_{i}\|h(y)\| A_{i}\right), D_{i}=h\left(B_{i} \| h(x \| y)\right)$, and $E_{i}=B_{i} \oplus h(x \| y)$.

(iii) $R C$ saves $\left\{C_{i}, D_{i}, E_{i}, h(),. h(y)\right\}$ into a smart card and sends to $U_{i}$ via a secure channel.

(iv) $U_{i}$ inputs $b$ into the smart card, and finally, $U_{i}$ has $\left\{C_{i}, D_{i}, E_{i}, b, h(),. h(y)\right\}$.

In the registration phase, we see that the author used common key $h(y)$, and this is dangerous because the adversary can exploit this to launch an impersonation attack if the smart card's information is leaked or stolen. Figure 1 describes all steps in this phase.
TABLE 1: Notations used in the scheme [11].

\begin{tabular}{lc}
\hline Notations & Description \\
\hline$U_{i}, S_{j}, R C$ & $i^{\text {th }}$ user, $j^{\text {th }}$ server, registration centre \\
$I D_{i}, P W_{i}$ & Identity and password of $U_{i}$ \\
$S I D_{j}, C I D_{i}$ & $S_{j}$ s identity, $U_{i}^{\prime}$ s dynamic identity \\
$x, y$ & Master key and secret value of $R C$ \\
$h(),. \oplus, \|$ & Hash function, XOR, and concatenation \\
$\Rightarrow / \longrightarrow$ & Secure/public channels \\
\hline
\end{tabular}

3.1.2. Login Phase. When logging into service, $U_{i}$ performs as follows:

(i) $U_{i}$ provides his/her smart card and inputs $I D_{i}$ and $P W_{i}$. Then, the smart card computes $A_{i}=h$ $\left(b \oplus P W_{i}\right)$ and $C_{i}^{*}=h\left(I D_{i}\|h(y)\| A_{i}\right)$ and checks if $C_{i}^{*}=C_{i}$. If this holds, $U_{i}$ continues. Otherwise, the smart card terminates the session.

(ii) The smart card randomly chooses values $N_{i}$ and computes $\quad P_{i j}=E_{i} \oplus\left(\mathrm{h} \quad(\mathrm{h}) \quad\left(S_{i} D_{j} \| h \quad(y)\right) \| N_{i}\right)$, $C I D_{i}=A_{i} \oplus\left(h(D)_{i}\left\|S I D_{j}\right\| N_{i}\right), M_{1}=h(P)_{i j}\left\|C I D_{i}\right\|$ $\left.D_{i} \| N_{i}\right)$, and $M_{2}=h\left(S I D_{j} \| h(y)\right) \oplus N_{i}$.

(iii) $U_{i}$ sends $\left\{P_{i j}, C I D_{i}, M_{1}, M_{2}\right\}$ to $S_{j}$ through a public channel.

At this phase, the random value $N_{i}$ can be easily computed because it is only protected by $h(y)$. This decreases the challenge from the user and makes the scheme unbalanced.

3.1.3. Authentication Phase. In this phase, the server also chooses the random value $N_{j}$ and only the valid user (who has $A_{i}$ ) can recompute this $N_{j}$ and send a correct response. Figure 2 describes all steps in this phase.

When $S_{j}$ receives $\left\{P_{i j}, C I D_{i}, M_{1}, M_{2}\right\}$ from $U_{i}, S_{j}$, and $U_{i}$, it performs the following steps:

(i) $S_{j}$ computes $N_{i}=h\left(S I D_{j} \| h(y)\right) \oplus M_{2}, E_{i}=P_{i j} \oplus h(h$ $\left.\left(S I D_{j} \| h(y)\right) \| N_{i}\right), B_{i}=E_{i} \oplus h(x \| y), D_{i}=h\left(B_{i} \| h\right.$ $(x \| y))$, and $A_{i}=C I D_{i} \oplus h\left(D_{i}\left\|S I D_{j}\right\| N_{i}\right)$.

(ii) $S_{j}$ computes $h\left(P_{i j}\left\|C I D_{i}\right\| D_{i} \| N_{i}\right)$ and compares it with $M_{1}$. If two values are unequal, $S_{j}$ rejects the login message and terminates the session. Otherwise, $S_{j}$ accepts login message. Then, $S_{j}$ randomly chooses $N_{j}$ and computes $M_{3}=h\left(D_{i}\left\|A_{i}\right\| N_{j} \| S I D_{j}\right)$ and $M_{4}=A_{i} \oplus N_{i} \oplus N_{j}$. Finally, $S_{j}$ sends $\left\{M_{3}, M_{4}\right\}$ to $U_{i}$ through a public channel.

(iii) When receiving $\left\{M_{3}, M_{4}\right\}$ from $S_{j}, U_{i}$ computes $N_{j}=A_{i} \oplus N_{i} \oplus M_{4}$ and $h\left(D_{i}\left\|A_{i}\right\| N_{j} \| S I D_{j}\right)$ and then compares it with $M_{3}$. If two values are unequal, $U_{i}$ rejects the message and terminates the session. Otherwise, $U_{i}$ successfully authenticates with $S_{j}$. Then, $U_{i}$ computes $M_{5}=h\left(D_{i}\left\|A_{i}\right\| N_{i} \| S I D_{j}\right)$ and sends $\left\{M_{5}\right\}$ to $S_{j}$ through a public channel.

(iv) $S_{j}$ computes $N_{i}=h\left(S I D_{j} \| h(y)\right) \oplus M_{2}, E_{i}=P_{i j} \oplus h(h$ $\left.\left(S I D_{j} \| h(y)\right) \| N_{i}\right), B_{i}=E_{i} \oplus h(x \| y), D_{i}=h\left(B_{i} \| h\right.$ $(x \| y))$, and $A_{i}=C I D_{i} \oplus h\left(D_{i}\left\|S I D_{j}\right\| N_{i}\right)$.

(v) When receiving $\left\{M_{5}\right\}$ from $U_{i}, S_{j}$ computes $h\left(D_{i} \|\right.$ $\left.A_{i}\left\|N_{i}\right\| S I D_{j}\right)$ and compares it with $M_{5}$. If two 


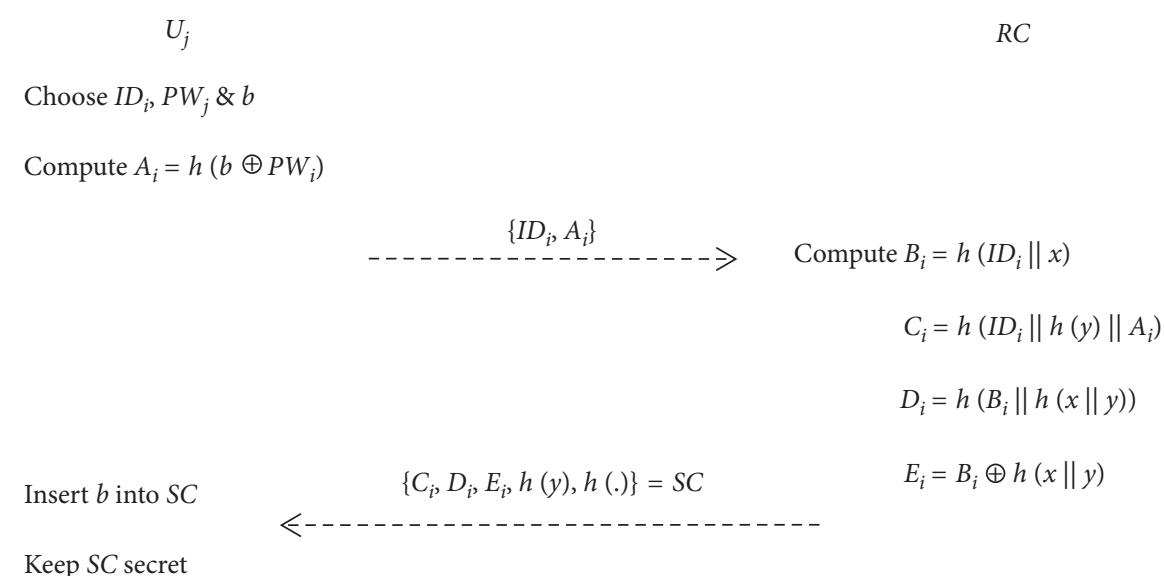

FIgURE 1: User registration phase of Li et al.'s scheme.

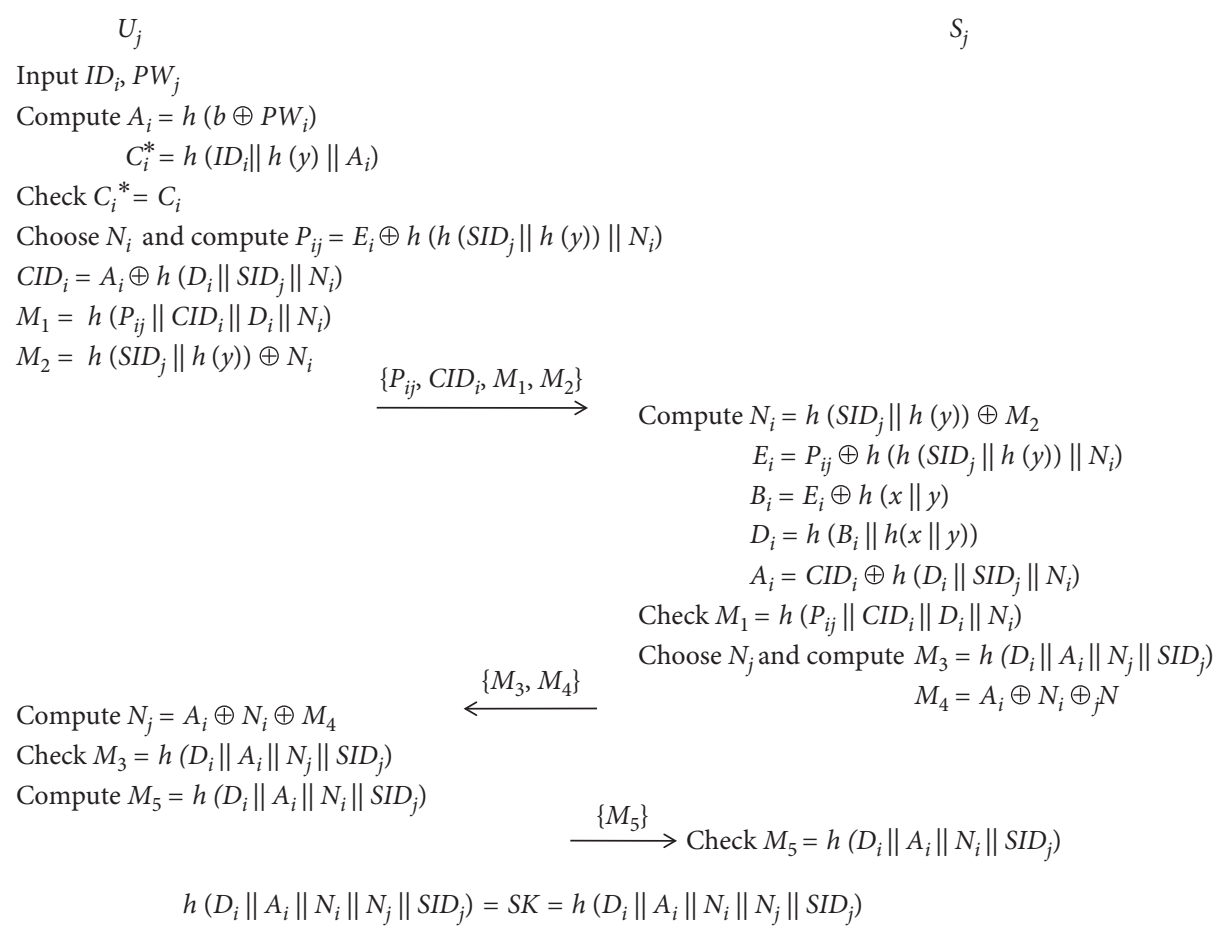

Figure 2: User authentication phase of Li et al.'s scheme.

values are equal, $S_{j}$ successfully authenticates with $U_{i}$ and authentication phase completes.

(vi) Next, $U_{i}$ and $S_{j}$ compute a common session key $S K=h\left(D_{i}\left\|A_{i}\right\| N_{i}\left\|N_{j}\right\| S I D_{j}\right)$ used to encrypt later transactions.

3.1.4. Password-Update Phase. This phase is performed when $U_{i}$ changes $P W_{i}$ into $P W_{\text {inew }}$ without interacting with $R C$ :

(i) $U_{i}$ inputs $I D_{i}$ and $P W_{i}$ and provides his/her smart card at the terminal.

(ii) The smart card computes $A_{i}=h\left(b \oplus P W_{i}\right)$ and $C_{i}^{*}=h\left(I D_{i}\|h(y)\| A_{i}\right)$ and checks if $C_{i}^{*}=C_{i}$. Is this does not hold, the smart card rejects password- update request. Otherwise, $U_{i}$ inputs $P W_{\text {inew }}$ and random number $b_{\text {new }}$.

(iii) The smart card computes $A_{\text {inew }}=h\left(b_{\text {new }} \oplus P W_{\text {inew }}\right)$ and $C_{\text {inew }}=h\left(I D_{i}\|h(y)\| A_{\text {inew }}\right)$.

(iv) Finally, the smart card replaces $C_{i}$ with $C_{\text {inew }}$ and terminates the session.

3.1.5. The Scheme's Cryptanalysis. If $U_{i}$ loses his/her smart card, it can result in impersonation attack. Furthermore, another attacker $U_{a}$ can exploit $S_{j}$ to guess the password through session key. Therefore, Li's scheme is also vulnerable to two-factor attack. Below are some steps to launch an impersonation and password-guessing attacks: 
(i) $U_{a}$ computes $P_{i j}=E_{i} \oplus h \quad\left(h \quad\left(S I D_{j} \| h \quad(y)\right) \| N_{i}\right)$, where $E_{i}$ is extracted from the smart card. $S I D_{j}, h$ $(y)$, and $N_{i}$ are easily computed by $U_{a}$ because they are public information.

(ii) Next $U_{a}$ computes $C I D_{i}=A_{i} \oplus h\left(D_{i}\left\|S I D_{j}\right\| N_{i}\right)$, $M_{1}=h\left(P_{i j}\left\|C I D_{i}\right\| D_{i} \| N_{i}\right)$, and $M_{2}=h\left(S I D_{j} \| h\right.$ $(y)) \oplus N_{i}$, where $A_{i}=h\left(b \oplus P W_{i}\right)$ is $U_{a}$ 's value and $D_{i}$ is extracted from $U_{i}^{\prime}$ 's smart card.

(iii) $U_{a}$ sends $\left\{P_{i j}, C I D_{i}, M_{1}, M_{2}\right\}$ to $S_{j}$. When receiving, $S_{j}$ will perform the following steps to verify.

(iv) $S_{j}$ extracts $N_{i}=h\left(S I D_{j} \| h(y)\right) \oplus M_{2}, E_{i}=P_{i j} \oplus h(h$ $\left.\left(S^{\prime} D_{j} \| h(y)\right) \| N_{i}\right), B_{i}=E_{i} \oplus h(x \| y), D_{i}=h\left(B_{i} \| h\right.$ $(x \| y))$, and $A_{i}=C I D_{i} \oplus h\left(D_{i}\left\|S I D_{j}\right\| N_{i}\right)$.

(v) $S_{j}$ sees $M_{1}=h\left(P_{i j}\left\|C I D_{i}\right\| D_{i} \| N_{i}\right)$, so $S_{j}$ randomly chooses $N_{j}$ and computes $M_{3}=h\left(D_{i}\left\|A_{i}\right\| N_{j} \| S I D_{j}\right)$ and $M_{4}=A_{i} \oplus N_{i} \oplus N_{j}$. $S_{j}$ sends $\left\{M_{3}, M_{4}\right\}$ to $U_{a}$.

(vi) When receiving $\left\{M_{3}, M_{4}\right\}, U_{a}$ computes $M_{5}=h$ $\left(D_{i}\left\|A_{i}\right\| N_{i} \| S I D_{j}\right)$ and sends to $S_{j}$.

(vii) $S_{j}$ sees $M_{5}=h\left(D_{i}\left\|A_{i}\right\| N_{i} \| S I D_{j}\right)$ and accepts $U_{a}$.

(viii )Finally, $U_{a}$ and $S_{j}$ compute a common session key $S K=h\left(D_{i}\left\|A_{i}\right\| N_{i}\left\|N_{j}\right\| S I D_{j}\right)$.

Because $E_{i}$ is extracted from $U_{i}$ 's smart card, the values $B_{i}$ and $D_{i}$ also belong to $U_{i}$. However, in Li's scheme, $A_{i}$ is separated from other values, so $U_{a}$ can exploit this limitation to insert his/her information. Furthermore, if $U_{a}$ captures previous transactions between $U_{i}$ and $S_{j}$, he/she will launch $U_{i}$ 's password-guessing attack. Assuming $U_{a}$ has $U_{i}^{\text {'s }} M_{5}=h$ $\left(D_{i}\left\|A_{i}\right\| N_{i} \| S I D_{j}\right)$, so $U_{a}$ can construct $h \quad\left(D_{i} \| h\right.$ $(b \oplus$ guess $\left.)\left\|N_{i}\right\| S I D_{j}\right)=M_{5}$ and use the password dictionary to search "guess" until success. Note that $U_{i}$ 's $N_{i}$ is easily found by computing $N_{i}=M_{2} \oplus h\left(S I D_{j} \| h(y)\right)$, in which $S I D_{j}$ and $h(y)$ are those $U_{a}$ easily computes.

3.2. $\mathrm{Qu}$ and Tan's Scheme. Qu and Tan's scheme [12] uses ECC, and it is secure against some popular kinds of attacks as they claimed. However, we will prove their scheme is vulnerable to impersonation attack. This scheme includes five phases: initialization, registration, login, authentication, and password-update phases. Table 2 presents some notations used in this scheme.

3.2.1. System Initialization. In this phase, the system initializes some parameters:

(i) $S$ chooses the elliptic curve $E_{P}(a, b)$ and base point $P$ with big prime order $n$

(ii) $S$ chooses $q_{S} \in[1, n-1]$ and computes the public key $Q_{S}=q_{S} \times P$

(iii) $S$ chooses three hash functions, $H_{1}(),. H_{2}($.$) , and H_{3}$ (.), described in Table 2

(iv) $S$ published $\left\{E_{P}(a, b), P, Q_{S}, H_{1}(),. H_{2}(),. H_{3}().\right\}$

In this phase, we see that $H_{1}($.$) is special because it$ receives any string and outputs a point belonging to the elliptic curve.
TABLE 2: Notations used in the scheme [12].

\begin{tabular}{lc}
\hline Notations & Description \\
\hline$S, U,\left(q_{S}, Q_{S}\right)$ & Server/user, key-pair of $S$ \\
$I D_{U}, P W_{U}$ & Identity and password of $U$ \\
$H_{1}$ & Hash function $\{0,1\}^{*} \longrightarrow \mathbb{G}_{p}$ \\
$H_{2}$ & Hash function $\mathbb{G}_{p} \times \mathbb{G}_{p} \longrightarrow \mathbb{Z}_{p}^{*}$ \\
$H_{3} \mid$ & Hash function $\{0,1\}^{*} \times \mathbb{G}_{p} \times \mathbb{G}_{p} \longrightarrow\{0,1\}^{k}$ \\
$r_{U}, r_{S}$ & Secret values of $U$ and $S$ \\
$\mathbb{F}_{p}, E_{P}\left(\mathbb{F}_{p}\right)$ & Finite field, elliptic curve defined over $\mathbb{F}_{p}$ \\
$\mathbb{G}$ & Group of points in $E_{P}\left(\mathbb{F}_{p}\right),|\mathbb{G}|=n \in \mathbb{P}$ \\
$P$ & Base point $P$ is a generator of $\mathbb{G}$ \\
\hline
\end{tabular}

3.2.2. Registration Phase. When registering, $U$ must follow following steps:

(i) $U$ chooses $I D_{U}, P W_{U}$, and random numbers $b_{U} \in[1$, $n-1]$ and then $U$ provides $I D_{U}$ and $H_{1}\left(P W_{U} \| b_{U}\right) \times$ $P$ to $S$ through a secure channel

(ii) When receiving $\left\{I D_{U}, H_{1}\left(P W_{U} \| b_{U}\right) \times P\right\}$ from $U, S$ computes $A I D_{U}=\left(q_{S}+1\right) \times H_{1}\left(P W_{U} \| b_{U}\right) \times P$ and $B I D_{U}=H_{2}\left(H_{1}\left(I D_{U}\right) \| H_{1}\left(P W_{U} \| b_{U}\right) \times P\right)$

(iii) $S$ saves $\left\{A I D_{U}, B I D_{U}\right\}$ into the smart card and then sends to $U$ via a secure channel

(iv) When receiving, $U$ inputs $b_{U}$ into the smart card. Finally, $U$ has $\left\{A I D_{U}, B I D_{U}, b_{U}\right\}$

At this phase, $S$ attaches $U$ 's personal information with $S$ 's master key $q_{S}$ to create the user's authentication key by using $H_{1}$ (.). Figure 3 describes all steps in this phase.

3.2.3. Login Phase. When $U$ logins into $S, U$ provides $I D_{U}$, $P W_{U}$, and his/her smart card into the terminal. Then, the smart card performs the following steps:

(i) The smart card computes $B I D_{U}^{\prime}=H_{2}\left(H_{1}\left(I D_{U}\right) \|\left(H_{1}\right.\right.$ $\left.\left.\left(P W_{U} \| b_{U}\right) \times P\right)\right)$ and checks if $B I D_{U}^{\prime}=B I D_{U}\left(B I D_{U}\right.$ is stored in the smart card). If this holds, $U$ provides correct information. Otherwise, the smart card will terminate the session.

(ii) $U$ randomly chooses $r_{U} \in[1, n-1]$ and computes $T I D_{U}=A I D_{U}-H_{1} \quad\left(P W_{U} \| b_{U}\right) \times P, \quad M=r_{U} \times Q_{S}$, $C I D_{U}=I D_{U} \times H_{2} \quad\left(M \| T I D_{U}\right), \quad D I D_{U}=M+H_{1}$ $\left(P W_{U} \| b_{U}\right) \times P$, and $E I D_{U}=H_{3} \quad\left(I D_{U}\|M\| R\right)$, where $R=r_{U} \times P$.

(iii) The smart card sends $M_{1}=\left\{C I D_{U}, D I D_{U}, E I D_{U}, R\right\}$ to $S$ through a public channel.

In this phase, identity is not attached with $U$ 's authentication key, so this is a weak point that another adversary can exploit to launch an impersonation attack. Figure 4 describes all steps in this phase and authentication phase.

3.2.4. Authentication Phase. When receiving the login message from $U, S$ performs as follows:

(i) $S \quad$ computes $\quad M^{\prime}=q_{S} \times R, \quad H_{1} \quad\left(P W_{U} \| b_{U}\right) \times$ $P=D I D_{U}-M^{\prime}, T I D_{U}^{\prime}=q_{S} \times H_{1}\left(P W_{U} \| b_{U}\right) \times P$, and $I D_{U}=C I D_{U} \oplus H_{2}\left(M^{\prime} \| T I D_{U}^{\prime}\right)$. Then, $S$ checks if $H_{3}$ 
Choose $I D_{U}, P W_{U} \& b_{U} \in[1, n-1]$
Compute $A_{i}=h\left(b \oplus P W_{i}\right)$

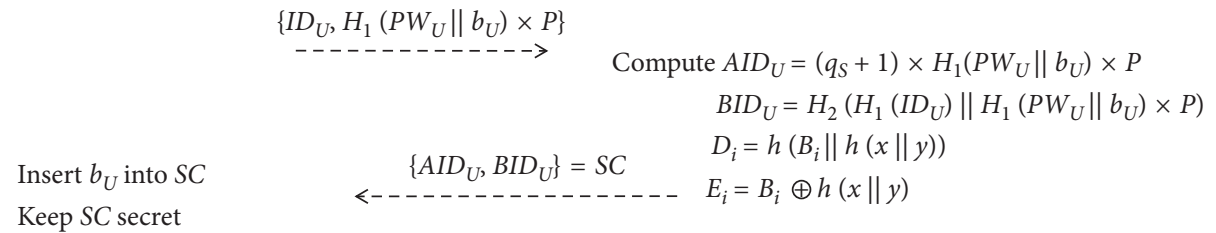

FIgURE 3: User registration phase of Qu et al.'s scheme.

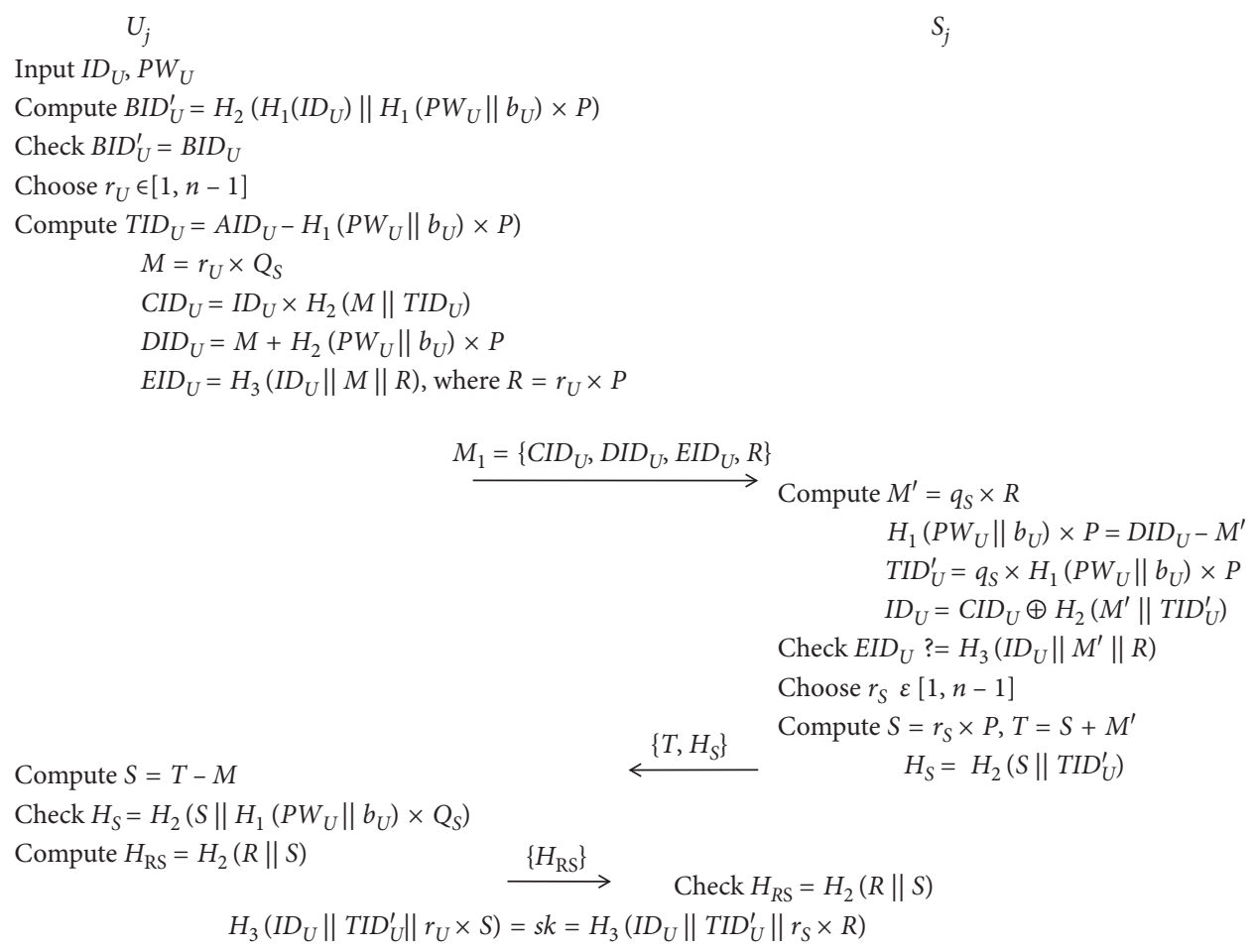

Figure 4: User authentication phase of Qu et al.'s scheme.

$\left(I D_{U}\left\|M^{\prime}\right\| R\right)=E I D_{U}$. If this holds, $S$ successfully authenticates with $U$. Otherwise, the session is terminated.

(ii) $S$ chooses $r_{S} \in[1, n-1]$ and then computes $S=r_{S} \times P, T=S+M^{\prime}$, and $H_{S}=H_{2}\left(S \| T I D_{U}^{\prime}\right)$.

(iii) $S$ sends $M_{2}=\left\{T, H_{S}\right\}$ to $U$ through a public channel.

(iv) When receiving $M_{2}=\left\{T, H_{S}\right\}$ from $S, U$ computes $S=T-M$ and $H_{S}^{\prime}=H_{2}\left(S \| H_{1}\left(P W_{U} \| b_{U}\right) \times Q_{S}\right)$ and checks if $H_{S}^{\prime}=H_{S}$. If this holds, $U$ successfully authenticates with $S$ and $U$ sends $M_{3}=\left\{H_{R S}\right\}$ to $S$, where $H_{R S}=H_{2}(R \| S)$. Otherwise, $U$ terminates the session.

(v) On receiving $M_{3}=\left\{H_{R S}\right\}$, $S$ computes $H_{R S}^{\prime}=H_{2}(R \| S)$ and checks if $H_{R S}^{\prime}=H_{R S}$. If this holds, $S$ and $U$ successfully authenticate each other. Otherwise, $S$ terminates the session. (vi) $U$ and $S$ compute common $S K=H_{3}\left(I D_{U}\left\|T I D_{U}\right\|\right.$ $\left.r_{U} \times S\right)=H_{3}\left(I D_{U}\left\|T I D_{U}^{\prime}\right\| r_{S} \times R\right)$.

3.2.5. Password-Update Phase. When receiving the login message from $U, S$ performs as follows:

(i) $U$ provides $I D_{U}, P W_{U}$, and the smart card at the terminal. Then, it computes $B I D_{U}^{\prime}=H_{2} \quad\left(H_{1}\right.$ $\left.\left(I D_{U}\right) \|\left(H_{1} \quad\left(P W_{U} \| b_{U}\right) \times P\right)\right)$ and checks if $B I D_{U}^{\prime}=B I D_{U}$. If this holds, $U$ can choose $P W_{U}^{\text {new }}$. Otherwise, the session is terminated.

(ii) The smart card computes $A I D_{U}^{\text {new }}=H_{1}\left(P W_{U} \|\right.$ $\left.b_{U}\right)^{-1} \times A I D_{U} \times H_{1}\left(P W_{U}^{\text {new }} \| b_{U}\right)$ and $B I D_{U}^{\text {new }}=H_{2}$ $\left(H_{1}\left(I D_{U}\right) \| H_{1}\left(P W_{U}^{\text {new }} \| b_{U}\right) \times P\right)$.

(iii) The smart card replaces $A I D_{U}$ and $B I D_{U}$ with $A I D_{U}^{\text {new }}$ and $B I D_{U}^{\text {new }}$. 
3.2.6. The Scheme's Cryptanalysis. If the user's identity is leaked, that user will be impersonated. Assuming another adversary is also a member. We call him/her $U_{a}$ with corresponding $\left\{A I D_{A}, B I D_{A}\right\}$ in his/her smart card. If $U_{a}$ knows victim's $I D_{U}, U_{a}$ performs the following steps to launch an impersonation attack:

(i) $U_{a}$ randomly chooses $r_{A} \in[1, n-1]$ and computes $R=r_{A} \times P$.

(ii) Next, $U_{a}$ extracts $T I D_{A}=A I D_{A}-H_{1}\left(P W_{A} \| b_{A}\right) \times P$, where $A I D_{A}, P W_{A}, b_{A}$, and $T I D_{A}$ are information in $U_{a}$ 's smart card.

(iii) $U_{a}$ computes $M=r_{A} \times Q_{S}, \quad C I D_{A}=I D_{U} \oplus H_{2} \quad(M \|$ $\left.T I D_{A}\right), D I D_{A}=M+H_{1}\left(P W_{A} \| b_{A}\right) \times P$, and $E I D_{A}=H_{3}$ $\left(I D_{U}\|M\| R\right)$. Then, $U_{a}$ sends $M_{1}=\left\{C I D_{A}, D I D_{A}\right.$, $\left.E I D_{A}, R\right\}$ to $S$.

(iv) When receiving $M_{1}, \quad S$ computes $M^{\prime}=q_{S} \times R=q_{S} \times r_{A} \times P=r_{A} \times Q_{S}, H_{1}\left(P W_{U} \| b_{U}\right) \times$ $P=D I D_{A}-M^{\prime}, T I D_{A}^{\prime}=q_{S} \times H_{1}\left(P W_{A} \| b_{A}\right) \times P$, and $I D_{U}=C I D_{A} \oplus H_{2}\left(M^{\prime} \| T I D_{A}^{\prime}\right)$.

(v) $S$ checks if $H_{3}\left(I D_{U}\left\|M^{\prime}\right\| R\right)=E I D_{A}$, and we see this condition holds.

(vi) $S$ randomly chooses $r_{S} \in[1, n-1]$, computes $S=r_{S} \times P, T=S+M^{\prime}$, and $H_{S}=H_{2}\left(S \| T I D_{A}^{\prime}\right)$, and sends $M_{2}=\left\{T, H_{S}\right\}$ to $U_{a}$.

(vii) On receiving $M_{2}, U_{a}$ computes $S=T-M$ and $H_{R S}=H_{2}(R \| S)$. Finally, $U_{a}$ sends $M_{3}=\left\{H_{R S}\right\}$ to $S$.

(viii) When receiving $M_{3}, S$ computes $H_{R S}{ }^{\prime}=H_{2}(R \| S)$ and see that $H_{R S}^{\prime}=H_{R S}$.

If the user's identity is leaked, he/she will be impersonated. The reason is that the user's identity is not attached with their secret information, for example, the authentication key $A I D_{U}$ is not attached with identity, or $B I D_{U}$ is only used for verification of the smart-card owner and does not take part in the authentication phase.

3.3. Amin and Biswas's Scheme. Amin and Biswas's scheme [13] uses ECC and biohashing, a special hash function overcoming the problem of sensitive input which exists in traditional hash function. In 2004, Jin et al. [27] proposed a remarkable improved biohashing function. Amin and Biswas's scheme includes four phases: registration, login, authentication, and password-update phases. Table 3 presents some notations used in this scheme.

3.3.1. Registration Phase. In this phase, $U_{i}$ chooses $I D_{i}, P W_{i}$, and biometrics $T_{i}$. Next, $U_{i}$ computes $A_{i}=h\left(I D_{i} \| P W_{i}\right)$ and $F_{i}=H\left(T_{i}\right)$ and sends $\left\{I D_{i}, A_{i}, F_{i}\right\}$ to $S$ through a secure channel. When receiving $\left\{I D_{i}, A_{i}, F_{i}\right\}$ from $U_{i}, S$ computes $W=h\left(I D_{S}\|x\| I D_{i}\right), B_{i}=h\left(I D_{i} \| A_{i}\right) \oplus W$, and $C I D_{i}=E N C_{x}$ $\left(I D_{i} \| R_{\text {ran }}\right)$ and sends a smart card including $\left\{F_{i}, A_{i}, B_{i}, C I D_{i}\right.$, $h(),. H()$.$\} back to U_{i}$ through a secure channel, where $I D_{S}$ is $S$ 's identity and $R_{\text {ran }}$ is the random number chosen by $S$. In this phase, $U_{i}$ can choose $I D_{i}$ and $P W_{i}$ easily guessed by password-guessing attack or identity-guessing attack. Figure 5 describes all steps in this phase.
TABLE 3: Notations used in the scheme [13].

\begin{tabular}{lc}
\hline Notations & Description \\
\hline$U_{i}, S$ & $i^{\text {th }}$ user, medical centre \\
$P W_{i}, I D_{i}, B_{i}$ & Password/identity/biometrics of $U_{i}$ \\
$p, q, \mathbb{F}_{p}$ & Two prime numbers, finite field \\
$E_{P}\left(\mathbb{F}_{p}\right)$ & EC in $\mathbb{F}_{p}: y^{2}=x^{3}+a x+b\left(a, b \in \mathbb{F}_{p}\right)$ and \\
$\mathbb{G}$ & $\delta=4 a^{3}+27 b^{2} \neq 0$ \\
$P$ & Group of points in $E_{P}\left(\mathbb{F}_{p}\right)$ \\
$a P$ & Base point of $\mathbb{G}$ with prime order $q$ \\
$x$ & Point multiplication $P$ \\
$\mathbb{Z}_{p}^{*}$ & Secret key of $S(1024$ bit $)$ \\
$h()$. & Multiplicative group \\
$H()$. & Hash function $\{0,1\}^{*} \longrightarrow \mathbb{Z}_{p}^{*}$ \\
$h_{1}()$. & Biohashing \\
$\oplus, \|, E N C / D E C$ & XOR, concatenation, encrypt $/$ decrypt \\
\hline
\end{tabular}

3.3.2. Login Phase. When $U_{i}$ successfully registers, $U_{i}$ performs as follows:

(i) $U_{i}$ provides the smart card with $T_{i}$, and then the smart card computes $F_{i}^{*}=H\left(T_{i}\right)$ and checks if $F_{i}^{*}=F_{i}\left(F_{i}\right.$ is stored in the smart card). If this condition holds, $U_{i}$ continues providing $I D_{i}$ and $P W_{i}$; otherwise, the scheme is terminated.

(ii) The smart card computes $A_{i}^{*}=h\left(I D_{i} \| P W_{i}\right)$ and checks if $A_{i}^{*}=A_{i}\left(A_{i}\right.$ is stored in the smart card). If this condition holds, the phase continues; otherwise, it is terminated.

(iii) $U_{i}$ randomly chooses $r_{i}$, computes $C_{1}=r_{i} \times P$, $W=B_{i} \oplus h\left(I D_{i} \| A_{i}^{*}\right), C_{2}=r_{i} \oplus W$, and $C_{4}=h\left(I D_{i} \|\right.$ $\left.r_{i} \| W\right)$, and sends $\left\{C_{2}, C_{4}, C I D_{i}\right\}$ to $S$ through a public channel.

In this phase, $U_{i}$ needs to use biometrics + password + identity to prove the smart-card owner. This method protects the user from impersonation attacks. Figure 6 describes all steps in this phase and the authentication phase.

3.3.3. Authentication Phase. When $S$ receives $\left\{C_{2}, C_{4}, C I D_{i}\right\}$ from $U_{i}, S$ and $U_{i}$ perform as follows:

(i) When receiving $\left\{C_{2}, C_{4}, C I D_{i}\right\}$ from $U_{i}, S$ decrypts $C I D_{i}$ by using $x$ and $S$ obtains $\left(I D_{i}^{*} \| R_{\text {ran }}\right)=D E C_{x}$ $\left(C I D_{i}\right)$. Then, $S$ computes $W=h\left(I D_{S}\|x\| I D_{i}^{*}\right)$, $r_{i}^{*}=C_{2} \oplus W, C_{1}^{*}=r_{i}^{*} \times P$, and $C_{4}^{*}=h\left(I D_{i}\left\|r_{i}^{*}\right\| W\right)$ and checks if $C_{4}^{*}=C_{4}\left(C_{4}\right.$ is stored in the smart card). If this condition holds, $S$ believes $U_{i}$ is the valid user.

(ii) $S$ randomly chooses $r_{j}$, computes $D_{1}=r_{j} \times P$, $S K=r_{j} \times C_{1}^{*}=r_{j} \times r_{i}^{*} \times P, \quad G_{1}=D_{1}+C_{1}^{*}, \quad L_{\mathrm{i}}=h$ $\left(I D_{i}^{*}\left\|h_{1} \quad\left(D_{1}\right)\right\| W\right), \quad$ and $\quad C I D_{i}^{\prime}=E N C_{x}$ $\left(I D_{i}^{*} \| R_{\text {ran }}^{\prime}\right)$, and sends $\left\{L_{i}, G_{1}, C I D_{i}^{\prime}\right\}$ to $U_{i}$ through a public channel.

(iii) When receiving $\left\{L_{i}, G_{1}, C I D_{i}^{\prime}\right\}$ from $S, U_{i}$ computes $D_{1}^{*}=G_{1}-C_{1}^{*}, L_{i}^{*}=h\left(I D_{i}\left\|h_{1}\left(D_{1}^{*}\right)\right\| W\right)$, and 


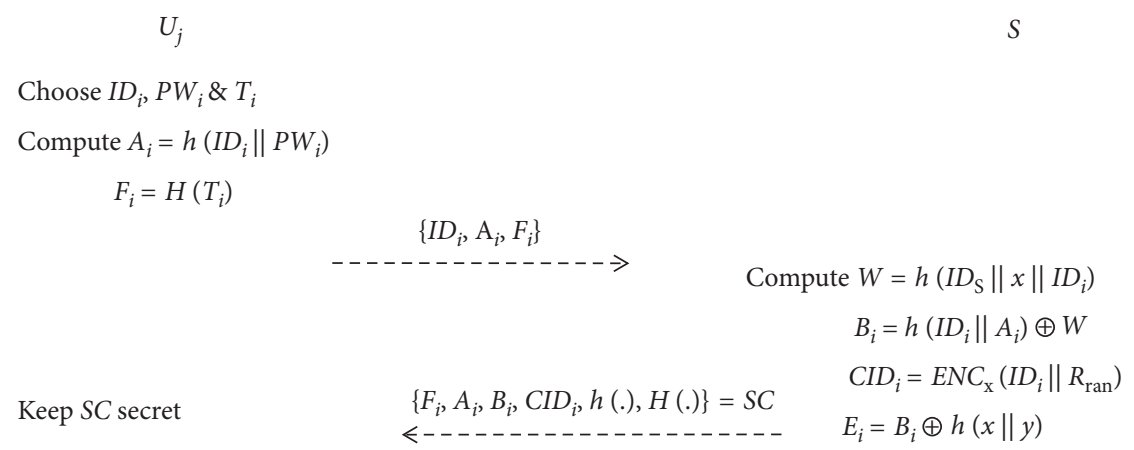

FIgURE 5: User registration phase of Amin et al.'s scheme.

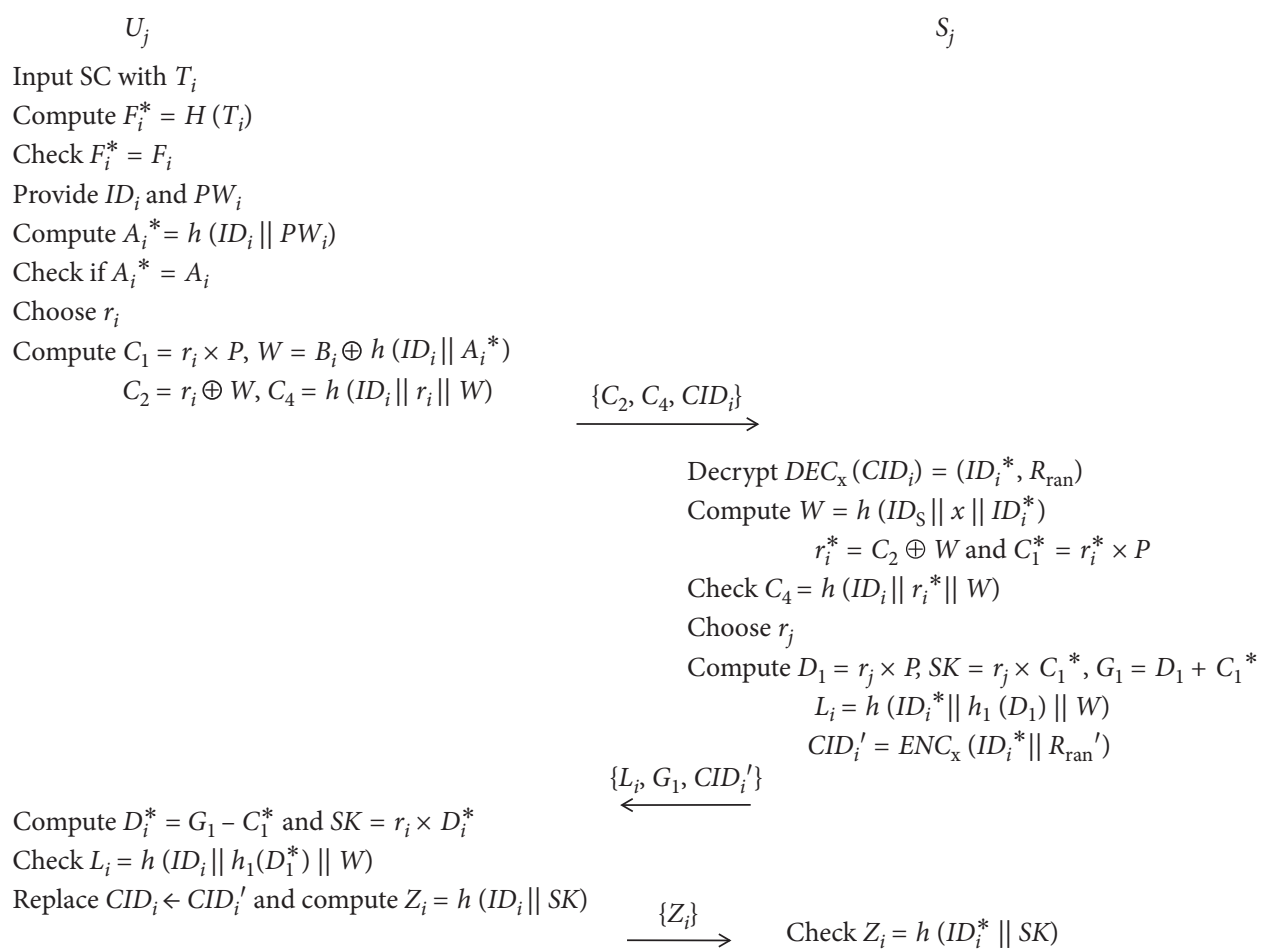

FIgURE 6: User authentication phase of Amin et al.'s scheme.

$S K=r_{i} \times D_{1}^{*}=r_{i} \times r_{j} \times P$ and checks if $L_{i}^{*}=L_{i}$. If this condition holds, $U_{i}$ believe $S$ is valid and $S K$ is a common session key of $U_{i}$ and $S$. After the successful authentication phase, $U_{i}$ replaces $C I D_{i}$ with $C I D_{i}^{\prime}$. Finally, $U_{i}$ computes $Z_{i}=h\left(I D_{i} \| S K\right)$ and sends to $S$ through a public channel.

(iv) When receiving $\left\{Z_{i}\right\}$ from $U_{i}, S$ computes $Z_{i}^{*}=h$ $\left(I D_{i}^{*} \| S K\right)$ and checks if $Z_{i}^{*}=Z_{i}$. If this condition holds, the authentication phase successfully completes.

In this phase, replacing $C I D_{i}$ after successfully authentication will enhance the user's privacy. Because each transaction has a different value, there is no way to know who is online, as well we cannot identify whether two transactions belong to one user.
3.3.4. Password-Update Phase. $U_{i}$ needs to successfully login if he/she wants to change the password. $U_{i}$ needs to provide $P W_{\text {inew }}$, and then his/her smart card computes $A_{\text {inew }}=h$ $\left(I D_{i} \| P W_{\text {inew }}\right)$ and $B_{\text {inew }}=h\left(I D_{i} \| A_{\text {inew }}\right) \oplus W$, where $W$ is the old value and replaces $\left(A_{i}, B_{i}\right)$ with $\left(A_{\text {inew }}, B_{\text {inew }}\right)$.

3.3.5. The Scheme's Cryptanalysis. If the master key is leaked, all previous exchanged messages between the user and server are also leaked. For example, if the key $x$ is leaked, the adversary stores previous message packages of the user and server, such as $\left\{C_{2}, C I D_{i}, C_{4}\right\}$ or $\left\{L_{i}, G_{1}, C I D_{i}^{\prime}\right\}$. The adversary will extract $I D_{i}$ by using $x$ to decrypt $C I D_{i}$, computes $W=h$ $\left(I D_{S}\|x\| I D_{i}\right)$ and $r_{i}=C_{2} \oplus W$. With $r_{i}$, the adversary computes $C_{1}=r_{i} \times P$ and $D_{1}=G_{1}-C_{1}$. From $r_{i}$ and $D_{i}$, the adversary finally computes $S K=r_{i} \times D_{1}$. 
3.4. Jangirala et al.'s Scheme. This scheme [18] uses hash function combined with random values, including four phases: registration, login, authentication, and passwordupdate phases. Because designed for multiserver environment, the registration centre constructs the master key $h(x \|$ $y$ ) for itself and the submaster key $h\left(S I D_{j} \| h(y)\right)$ for each service provider. Notations used in this scheme are in Table 1.

\subsubsection{Registration Phase. $U_{i}$ registers with $R C$ as follows:}

(i) $U_{i}$ chooses $I D_{i}, P W_{i}$, and random value $b$ and computes $A_{i}=h\left(I D_{i} \oplus b \oplus P W_{i}\right)$. Then, $U_{i}$ sends $I D_{i}$ and $A_{i}$ to $R C$ through a secure channel.

(ii) On receiving $\left\{I D_{i}, A_{i}\right\}$ from $U_{i}, R C$ computes $B_{i}=h$ $\left(A_{i} \| x\right), C_{i}=h\left(I D_{i}\|h(y)\| A_{i}\right), D_{i}=h\left(B_{i} \| h(x \| y)\right)$, and $E_{i}=B_{i} \oplus h(x \| y)$.

(iii) RC saves $\left\{C_{i}, D_{i}, E_{i}, h(),. h(y)\right\}$ into a smart card and sends to $U_{i}$ via a secure channel.

(iv) $U_{i}$ computes $L_{i}=b \oplus h\left(I D_{i} \| P W_{i}\right)$. Then, $U_{i}$ inserts $L_{i}$ into the smart card, and finally, $U_{i}$ has $\left\{C_{i}, D_{i}, E_{i}\right.$, $\left.L_{i}, h(),. h(y)\right\}$.

In the registration phase, we see that their scheme encrypts $b$ with $h\left(I D_{i} \| P W_{i}\right)$. This prevents some kinds of privileged insider attacks. Figure 7 describes all steps in this phase.

3.4.2. Login Phase. This phase sends $U_{i}$ 's login request to $S_{j}$ as follows:

(i) $U_{i}$ inserts his/her smart card and inputs $I D_{i}$ and $P W_{i}$. Then, the smart card computes $b=L_{i} \oplus h$ $\left(I D_{i} \| P W_{i}\right), \quad A_{i}=h \quad\left(I D_{i} \oplus b \oplus P W_{i}\right), \quad$ and $\quad C_{i}^{*}=h$ $\left(I D_{i}\|h(y)\| A_{i}\right)$ and checks if $C_{i}^{*}=C_{i}$. If this holds, $U_{i}$ continues. Otherwise, the smart card terminates the session.

(ii) The smart card randomly chooses values $N_{i}$ and computes $\quad P_{i j}=E_{i} \oplus h \quad\left(h \quad\left(S I D_{j} \| h \quad(y)\right) \| N_{i}\right)$, $C I D_{i}=A_{i} \oplus h \quad\left(D_{i}\left\|S I D_{j}\right\| N_{i}\right), \quad M_{1}=h \quad\left(P_{i j}\left\|C I D_{i}\right\|\right.$ $\left.A_{i} \| N_{i}\right)$, and $M_{2}=h\left(S I D_{j} \| h(y)\right) \oplus N_{i}$.

(iii) $U_{i}$ sends $\left\{P_{i j}, C I D_{i}, M_{1}, M_{2}\right\}$ to $S_{j}$ through a public channel.

At this phase, random value $N_{i}$ can be easily known by the adversary because it is only protected by $h(y)$. Furthermore, if the user's smart card is leaked, the adversary can compute his/her $D_{i}$ and discover what the user did in previous session corresponding to $N_{i}$.

3.4.3. Authentication Phase. When $S_{j}$ receives $\left\{P_{i j}, C I D_{i}, M_{1}\right.$, $M_{2}$ \} from $U_{i}, S_{j}$ verifies $U_{i}$ 's login message as follows:

(i) $S_{j}$ computes $N_{i}=h\left(S I D_{j} \| h(y)\right) \oplus M_{2}, E_{i}=P_{i j} \oplus h(h$ $\left.\left(S I D_{j} \| h(y)\right) \| N_{i}\right), B_{i}=E_{i} \oplus h(x \| y), D_{i}=h\left(B_{i} \| h\right.$ $(x \| y))$, and $A_{i}=C I D_{i} \oplus h\left(D_{i}\left\|S I D_{j}\right\| N_{i}\right)$.

(ii) $S_{j}$ computes $h\left(P_{i j}\left\|C I D_{i}\right\| A_{i} \| N_{i}\right)$ and compares it with $M_{1}$. If two values are not matched, $S_{j}$ rejects the login message and terminates the session. Otherwise, $S_{j}$ accepts the login message. Then, $S_{j}$ randomly chooses $N_{j}$ and computes $S K_{i j}=h\left(h\left(B_{i} \| h(x \| y)\right) \|\right.$ $\left.A_{i}\right), \quad M_{3}=h \quad\left(S K_{i j}\left\|A_{i}\right\| S I D_{j} \| N_{j}\right), \quad$ and $M_{4}=S K_{i j} \oplus N_{j} \oplus N_{j}$. Finally, $S_{j}$ sends $\left\{M_{3}, M_{4}\right\}$ to $U_{i}$ through a public channel.

(iii) When receiving $\left\{M_{3}, M_{4}\right\}$ from $S_{j}, U_{i}$ computes $S K_{i j}=h\left(D_{i} \| A_{i}\right), N_{j}=S K_{i j} \oplus M_{4}$, and $h\left(S K_{i j}\left\|A_{i}\right\|\right.$ $\left.S I D_{j} \| S I D_{j}\right)$ and then compares it with $M_{3}$. If two values are not matched, $U_{i}$ rejects the message and terminates the session. Otherwise, $U_{i}$ successfully authenticates with $S_{j}$. Then, $U_{i}$ computes $M_{5}=h$ $\left(S K_{i j}\left\|A_{i}\right\| S I D_{j}\left\|N_{i}\right\| N_{j}\right)$ and sends $\left\{M_{5}\right\}$ to $S_{j}$ through a public channel.

(iv) When receiving $\left\{M_{5}\right\}$ from $U_{i}, S_{j}$ computes $h\left(S K_{i j} \|\right.$ $\left.A_{i}\left\|S I D_{j}\right\| N_{i} \| N_{j}\right)$ and compares it with $M_{5}$. If two values are equal, $S_{j}$ successfully authenticates with $U_{i}$.

(v) Next, $U_{i}$ and $S_{j}$ compute a common session key $S K e y_{i j}=h \quad\left(S K_{i j}\left\|A_{i}\right\| S I D_{j}\left\|N_{i}\right\| D_{i} \| N_{j}\right)$ used to encrypt later transactions. Also, $S_{j}$ chooses the random value $N_{j}$ and only the valid user (who has $D_{i}$ and $A_{i}$ ) can extract this $N_{j}$ and send correct response. Figure 8 describes all steps in this phase.

3.4.4. Password-Update Phase. This phase is performed when $U_{i}$ changes $P W_{i}$ into $P W_{\text {inew }}$ without interacting with $R C$ :

(i) $U_{i}$ provides his/her smart card at the terminal and inputs $I D_{i}$ and $P W_{i}$.

(ii) The smart card computes $b^{*}=L_{i} \oplus h\left(I D_{i} \| P W_{i}\right)$, $A_{i}^{*}=h \quad\left(I D_{i} \oplus b^{*} \oplus P W_{i}\right), \quad$ and $\quad C_{i}^{*}=h \quad\left(I D_{i} \| h\right.$ $\left.(y) \| A_{i}^{*}\right)$ and checks if $C_{i}^{*}=C_{i}$. Is this does not hold, the smart card rejects and terminates the password-update-request session. Otherwise, $U_{i}$ inputs $P W_{\text {inew }}$.

(iii) The smart card computes $A_{i}^{\text {new }}=h$ $\left(I D_{i} \oplus b^{*} \oplus P W_{i}^{\text {new }}\right)$ and $C_{i}^{\text {new }}=h \quad\left(I D_{i}\left\|A_{i}^{\text {new }}\right\| h\right.$ $(y))$.

(iv) Finally, the smart card replaces $C_{i}$ with $C_{i}^{\text {new }}$ and $L_{i}$ with $L_{i}^{\text {new }}$, where $L_{i}^{\text {new }}=b^{*} \oplus h\left(I D_{i} \| P W_{i}^{\text {new }}\right)$.

3.4.5. The Scheme's Cryptanalysis. If another $U_{i}$ 's smart card leaks information $\left\{C_{i}, D_{i}, E_{i}, h(y), h().\right\}$ and the adversary $U_{a}$ is another valid user, $U_{a}$ can launch an impersonation attack as follows:

(i) $U_{a}$ computes $P_{i j}=E_{i} \oplus h\left(h\left(S I D_{j} \| h(y)\right) \| N_{a}\right)$, where $N_{a}$ is random value chosen by $U_{a}$

(ii) Then, $U_{a}$ computes $C I D_{i}=A_{a} \oplus h\left(D_{i}\left\|S I D_{j}\right\| N_{a}\right)$, $M_{1}=h\left(P_{i j}\left\|C I D_{i}\right\| A_{a} \| N_{a}\right)$, and $M_{2}=h\left(S I D_{j} \| h\right.$ $(y)) \oplus N_{a}$, where $A_{a}$ belongs to $U_{a}$

(iii) Next, $U_{a}$ sends $\left\{P_{i j}, C I D_{i}, M_{1}, M_{2}\right\}$ to $S_{j}$

(iv) Once receiving these messages, $S_{j}$ computes $N_{a}=h$ $\left(S I D_{j} \| h(y)\right) \oplus M_{2}, E_{i}=P_{i j} \oplus h\left(h\left(S I D_{j} \| h(y)\right) \| N_{a}\right)$, $B_{i}=E_{i} \oplus h(x \| y)$, and $D_{i}=h\left(B_{i} \| h(x \| y)\right)$ 
$U_{j} \quad R C$

Choose $I D_{i}, P W_{j}$ and $b$

Compute $A_{i}=h\left(I D_{i} \oplus b \oplus P W_{i}\right)$

$$
\begin{array}{cc}
\left\{I D_{i}, A_{i}\right\} & \\
& \text { Compute } B_{i}=h\left(A_{i} \| x\right) \\
C_{i}=h\left(I D_{i}\|h(y)\| A_{i}\right) \\
D_{i}=h\left(B_{i} \| h(x \| y)\right) \\
\quad E_{i}=B_{i} \oplus h(x \| y) \\
\left\{C_{i}, D_{i}, E_{i}, h(y), h(.)\right\}=S C \quad
\end{array}
$$

Insert $L_{i}=b \oplus h\left(I D_{i} \| P W_{i}\right)$ into $S C$

Keep $S C$ secret

FIgURE 7: User authentication phase of Jangirala et al.'s scheme.

$$
\begin{aligned}
& U_{j} \\
& \text { Input } I D_{i}, P W_{j} \\
& \text { Compute } b=L_{i} \oplus h\left(I D_{i} \| P W_{i}\right) \\
& A_{i}=h\left(I D_{i} \oplus b \oplus P W_{i}\right) \\
& \text { Check } C_{i}^{*}=C_{i} \\
& C_{i}^{*}=h\left(I D_{i}\|h(y)\| A_{i}\right) \\
& \text { Choose } N_{i} \text { and compute } P_{i j}=E_{i} \oplus h\left(h\left(S I D_{j} \| h(y)\right) \| N_{i}\right) \\
& C I D_{i}=A_{i} \oplus h\left(D_{i}\left\|S I D_{j}\right\| N_{i}\right) \\
& M_{1}=h\left(P_{i j}\left\|C I D_{i}\right\| A_{i} \| N_{i}\right) \\
& M_{2}=h\left(\operatorname{SID}_{j} \| h(y)\right) \oplus N_{i} \\
& \stackrel{\left\{P_{i j}, C I D_{i}, M_{1}, M_{2}\right\}}{\longrightarrow} \\
& \text { Compute } N_{i}=h\left(S I D_{j} \| h(y)\right) \oplus M_{2} \\
& E_{i}=P_{i j} \oplus h\left(h\left(S D_{j} \| h(y)\right) \| N_{i}\right) \\
& B_{i}=E_{i} \oplus h(x \| y) \\
& D_{i}=h\left(B_{i} \| h(x \| y)\right) \\
& A_{i}=C I D_{i} \oplus h\left(D_{i}\left\|S I D_{j}\right\| N_{i}\right) \\
& \text { Check } M_{1}=h\left(P_{i j}\left\|C I D_{i}\right\| A_{i} \| N_{i}\right) \\
& \text { Choose } N_{j} \text { and compute } S K_{i j}=h\left(h\left(B_{i} \| h(x \| y)\right) \| A_{i}\right) \\
& \text { Compute } S K_{i j}=h\left(D_{i} \| A_{i}\right) \text { and } N_{j}=S K_{i j} \oplus M_{4} \quad \stackrel{\left\{M_{3}, M_{4}\right\}}{\longleftarrow} \quad \begin{array}{l}
M_{3}=h\left(S K_{i j}\left\|A_{i}\right\| S I D_{j} \| N_{j}\right) \\
M_{4}=S K_{i j} \oplus N_{j}
\end{array} \\
& \text { Check } M_{3}=h\left(S K_{i j}\left\|A_{i}\right\| S I D_{j} \| N_{j}\right) \\
& \text { Compute } M_{5}=h\left(S K_{i j}\left\|A_{i}\right\| S I D_{j}\left\|N_{i}\right\| N_{j}\right) \quad \stackrel{\left\{M_{5}\right\}}{\longrightarrow} \text { Check } M_{5}=h\left(S K_{i j}\left\|A_{i}\right\| S I D_{j}\left\|N_{i}\right\| N_{j}\right) \\
& h\left(S K_{i j}\left\|A_{i}\right\| S I D_{j}\left\|N_{i}\right\| D_{i} \| N_{j}\right)=S K e y_{i j}=h\left(S K_{i j}\left\|A_{i}\right\| S I D_{j}\left\|N_{i}\right\| D_{i} \| N_{j}\right)
\end{aligned}
$$

FIGURE 8: User authentication phase of Jangirala et al.'s scheme.

(v) Next $S_{j}$ computes $A_{a}=C I D_{i} \oplus h\left(D_{i}\left\|S I D_{j}\right\| N_{a}\right)$ and sees that $M_{1}=h\left(P_{i j}\left\|C I D_{i}\right\| A_{a} \| N_{a}\right)$

(vi) $S_{j}$ chooses $N_{j}$ and computes $S K_{i j}=h\left(h\left(B_{i} \| h(x \|\right.\right.$ $\left.y)) \| A_{a}\right), \quad M_{3}=h \quad\left(S K_{i j}\left\|A_{a}\right\| S I D_{j} \| N_{j}\right), \quad$ and $M_{4}=S K_{i j} \oplus N_{j}$

(vii) Then, $S_{j}$ sends $\left\{M_{3}, M_{4}\right\}$ to $U_{a}$

(viii) Once receiving these messages from $S_{j}, U_{a}$ computes $S K_{i j}=h\left(D_{i} \| A_{a}\right)$ and $N_{j}=S K_{i j} \oplus M_{4}$ and sends $M_{5}$ to $S_{j}$, where $M_{5}=h\left(S K_{i j}\left\|A_{a}\right\| S I D_{j}\left\|N_{a}\right\| N_{j}\right)$.

(ix) Once receiving $M_{5}$ from $U_{a}, S_{j}$ sees that $M_{5}=h$ $\left(S K_{i j}\left\|A_{a}\right\| S I D_{j}\left\|N_{a}\right\| N_{j}\right)$ and computes the session key $S K e y_{i j}=h\left(S K_{i j}\left\|A_{a}\right\| S I D_{j}\left\|N_{a}\right\| D_{i} \| N_{j}\right)$

Clearly, $U_{a}$ successfully authenticates with $S_{j}$ without knowing the user's identity and password.
3.5. Han et al.'s Scheme. This scheme [19] uses the fuzzy extractor to process the user's biometrics, including four phases: registration, login, authentication, and passwordupdate phases. With symmetric encryption, this scheme truly has strong user anonymity because the adversary cannot know if two login sessions are belonged to the same user. Some notations used in this scheme are in Table 4.

3.5.1. Registration Phase. In the registration phase, we see that their scheme generates $\langle R, P\rangle$ from the user's biometrics with the fuzzy extractor. Furthermore, the user's dynamic identity is made by the server by using the encryption scheme. Figure 9 describes all steps in this phase.

Firstly, the user chooses $I D, P W$, biometrics $B$, and random value $r$. Then, the fuzzy extractor generates $\langle R, P\rangle$ 
TABle 4: Notations used in the scheme [19].

\begin{tabular}{lc}
\hline Notations & Description \\
\hline$U, S$ & User/patient, telecare server \\
$P W, I D, B$ & Password/identity/biometrics of $U$ \\
$s$ & Private key of server \\
$S K$ & Session key between $U$ and $S$ \\
$h()$. & Cryptographic one-way hash function \\
$E n c_{x}(.) / D e c_{x}()$. & Symmetric encryption scheme \\
$G e n$ & Probabilistic generation algorithm \\
$\operatorname{Rep}$ & Probabilistic reproduction algorithm \\
$\oplus, \|, T_{n}$ & XOR, concatenation, Chebyshev operation \\
\hline
\end{tabular}

from $B$ and the user computes $A=h(P W \| R) \oplus r$. Next, the user sends $\{I D, A\}$. Once receiving the user's messages, the server computes $A I D=h(I D \| s), K=h(A I D), V^{\prime}=A I D \oplus A$, and $C I D=E n c_{s}(I D \| a)$, where $a$ is chosen randomly by and $s$ is private key of the server. Then, the server sends $S C=\{K$, $\left.V^{\prime}, C I D, h().\right\}$ to the user. Once receiving the server's message, the user computes $V=V^{\prime} \oplus A \oplus h(I D\|P W\| R)$ and replaces $V^{\prime}$ by $V$. The user inserts $P$ in $S C$ and keeps it secret.

3.5.2. Login Phase. The user sends inserts $S C$ into the terminal and enters $I D, P W$, and $B^{\prime}$ similar to $B$. Then, $S C$ performs as follows:

(i) SC performs $\operatorname{Rep}\left(B^{\prime}, P\right)$ to generate $R$ and computes $A I D=V \oplus h(I D\|P W\| R)$

(ii) $S C$ checks if $K=h(A I D)$. If this holds, go to next step

(iii) SC generates a nonce $u$ and computes $X=T_{u}(A I D)$ and $V_{1}=h\left(I D\|X\| C I D \| T_{1}\right)$

(iv) $S C$ transmits $\left\{C I D, X, V_{1}, T_{1}\right\}$ to the server

At this phase, the user needs to recreate the $R$ value by providing correct his/her biometrics.

3.5.3. Authentication Phase. When receiving the login message from the user, $S$ verifies the login message as follows:

(i) $S$ checks if $\left|T_{c}-T_{1}\right| \leq \delta T$, where $T_{c}$ is receiving time. If this holds, $S$ retrieves ID by computing $\operatorname{Dec}_{s}$ (CID) with the private key $s$.

(ii) Then, $S$ computes $A I D=h(I D \| s)$ and checks if $V_{1}=h\left(I D\|X\| C I D \| T_{1}\right)$. If this holds, $S$ generates random values $a^{\prime}$ and $v$.

(iii) Then, $S$ computes $C I D^{\prime}=E n c_{s}\left(I D \| a^{\prime}\right), S K=h\left(T_{v}\right.$ $(X)), Y=T_{v}(A I D)$, and $V_{2}=h\left(C I D^{\prime}\|Y\| S K \| T_{2}\right)$, where $T_{2}$ is current time.

(iv) Then, $S$ sends $\left\{C I D^{\prime}, Y, V_{2}, T_{2}\right\}$ to the user.

(v) Once receiving messages from the server, $S C$ checks $T_{2}$ and calculates $S K=h\left(T_{u}(Y)\right)$.

(vi) Then, $S C$ checks if $V_{2}=h\left(C I D^{\prime}\|Y\| S K \| T_{2}\right)$. If this holds, $S C$ replaces $C I D$ with $C I D^{\prime}$, computes $V_{3}=h\left(S K \| T_{3}\right)$, and sends $\left\{V_{3}, T_{3}\right\}$ to $S$. (vii) Once receiving messages, the server checks $T_{3}$ and verifies if $V_{3}=h\left(S K \| T_{3}\right)$. If this holds, the user and server successfully authenticate to each other and accept $S K$ as a session key.

This scheme is completely dependent on random values $u$ and $v$, and this is vulnerable to known session-specific temporary information attack. Figure 10 describes all steps in this phase.

3.5.4. Password-Update Phase. This phase is performed when $U$ changes $P W$ into $P W^{\text {new }}$ without interacting with $S$ :

(i) $U$ inserts his/her smart card into the terminal and inputs $I D, P W$, and $B^{\prime}$. SC executes Rep $\left(B^{\prime}, P\right)=R$ and computes $A I D=V \oplus h(I D\|P W\| R)$.

(ii) $S C$ checks if $h(A I D)=K$. If this holds, $S C$ allows the change request.

(iii) $U$ inputs $P W^{\text {new }}$ and $B^{\text {new }}$, and then $S C$ computes $<R^{\text {new }}, P^{\text {new }}>=\operatorname{Gen}\left(B^{\text {new }}\right)$ and $V^{\text {new }}=V \oplus h(I D \|$ $P W \| R) \oplus h\left(I D\left\|P W^{\text {new }}\right\| R^{\text {new }}\right)$.

(iv) Finally, $S C$ replaces $V$ by $V^{\text {new }}$.

3.5.5. The Scheme's Cryptanalysis. If another session leaks the random value $v$, the adversary can exploit to reattack the user and know previous messages transmitted between the user and server. For example, the adversary $U_{a}$ obtains $\left\{C I D, X, V_{1}, T_{1}\right\}$, $\left\{C I D^{\prime}, Y, V_{2}, T_{2}\right\}$, and $\left\{V_{3}, T_{3}\right\}$ with the random value $v$ leaked, and $U_{a}$ can launch an impersonation attack as follows:

(i) If $U$ sends the new login message $\left\{C I D^{\prime}, X^{\prime}, V_{1}^{\prime}, T_{1}^{\prime}\right\}$ to $S, U_{a}$ can block this message.

(ii) $U_{a}$ generates random $C I D^{\prime \prime}$.

(iii) Then, $U_{a}$ computes $S K^{\prime}=h\left(T_{v}\left(X^{\prime}\right)\right)$ and $V_{2}^{\prime}=h$ $\left(C I D^{\prime \prime}\|Y\| S K^{\prime} \| T_{2}^{\prime}\right)$, where $T_{2}^{\prime}$ is current time and $Y$ is previous value of $U$ and $S$.

(iv) Then, $U_{a}$ sends $\left\{C I D^{\prime \prime}, Y, V_{2}^{\prime}, T_{2}^{\prime}\right\}$ to $U$.

(v) Once receiving the message from $U_{a}, U$ checks $T_{2}^{\prime}$. If this holds, $U$ computes $S K^{\prime}=h\left(T_{u^{\prime}}(Y)\right)$, where $u^{\prime}$ is a random value chosen by $U$.

(vi) $U$ checks if $V_{2}^{\prime}=h\left(C I D^{\prime \prime}\|Y\| S K^{\prime} \| T_{2}^{\prime}\right)$. We see this holds and $U$ sends $V_{3}^{\prime}=h\left(S K^{\prime} \| T_{3}^{\prime}\right)$ to $U_{a}$.

Clearly, the adversary can reuse this random value $v$ to reattack the user many times. The main reason is that CID is what the user does not know.

3.6. Proposed Scheme. In Section 3, we review some typical schemes using many approaches such as Chebyshev polynomial or elliptic curve cryptosystem in various environments. Although these schemes are well designed with some interesting primitives, such as fuzzy extractor or symmetric encryption scheme, they are still vulnerable to some typical kinds of attacks, such as password-guessing or impersonation. Indeed, there are still interesting schemes [17, 20], but they are designed with three-party participation different with two-party participation of the proposed scheme. 
User Server

Choose ID, $P W, B, r$

$(s)$

FIGURE 9: User registration phase of Han et al.'s scheme.

User

Input $I D, P W, B^{\prime}$

Compute $\operatorname{Rep}\left(B^{\prime}, P\right)=R$

$A I D=V \oplus h(I D\|P W\| R)$

Check $h(A I D)=K$

Choose $u$ and compute $X=T_{u}$ (AID)

$V_{1}=h\left(I D\|X\| C I D \| T_{1}\right)$

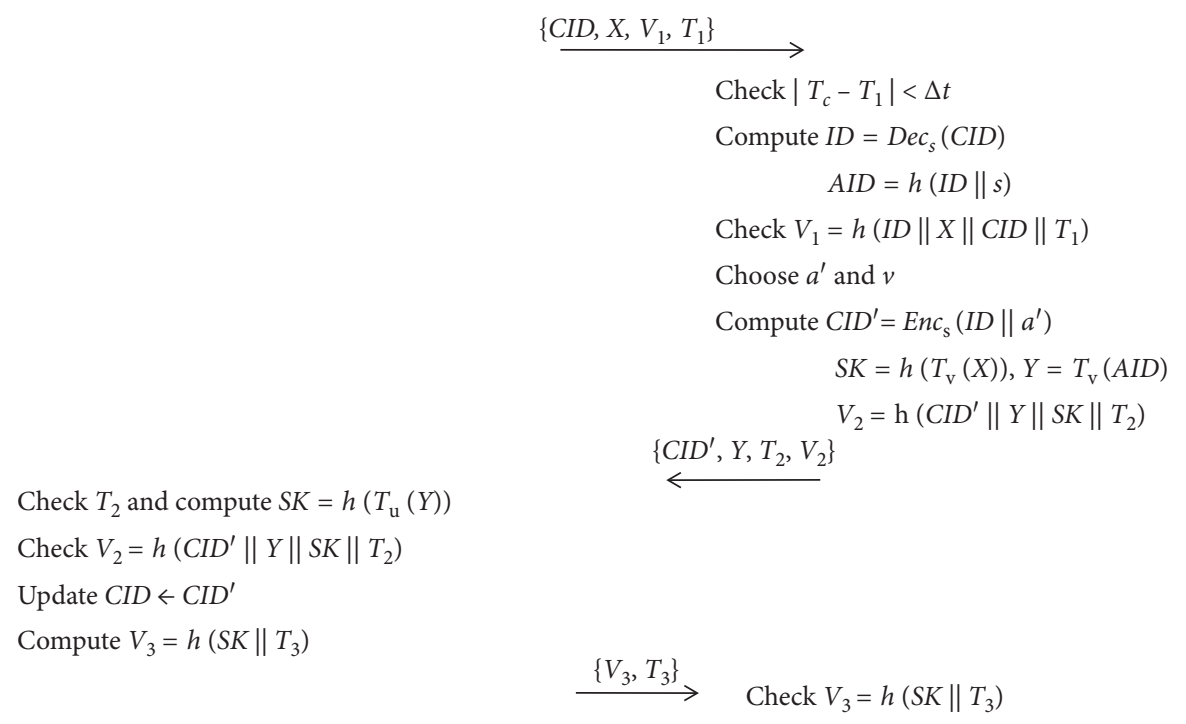

FIGURE 10: User authentication phase of Han et al.'s scheme.

Therefore, we temporarily do not consider in this paper. Figure 11 shows our architecture of participation between registration centre $(R C)$, servers $\left(S_{j}\right)$, and users $\left(U_{X}\right)$, where the keys of servers and users are created by $R C$.

With this architecture in Figure 11, we can deploy a $R C$ to centralize all medical servers. Also, the users easily find the medical services suitable for them. This section presents the phases in our proposed scheme. Our scheme uses Chebyshev polynomial in multiserver environment with two-party participation, including five phases: initialization, registration (server + user), authentication, and passwordupdate phases. Some notations used in our scheme are in Table 5.
3.6.1. System Initialization. $R C$ chooses the big prime number $p \in \mathbb{P} k$-bit and a $q_{R C}$. Then, $R C$ chooses $H_{0}:\{0,1\}^{*} \longrightarrow$ $\{0,1\}^{\mathrm{k}} . R C$ publishes $\left\{T(),. H_{0}(), p.\right\}$ and keeps $q_{R C}$ secret.

3.6.2. Server Registration Phase. In this phase, $S_{j}$ provides $S I D_{j}$ to $R C$ through a secure channel. $R C$ chooses $r_{j}$ and computes $A S I D_{j}=T_{q_{R C}}\left(H_{0}\left(S I D_{j} \| r_{j}\right)\right) \bmod p$ and then returns $\left\{r_{j}, S I D_{j}, A S I D_{j}, H_{0}().\right\}$ to $S_{j}$. Figure 12 shows the steps in this phase.

In this phase, each server $S_{j}$ has unique master key $A S I D_{j}$ produced by $R C$. $R C$ must keep the pair $<r_{j}, S I D_{j}>$ for subsequent retrieval and the user's registration. 


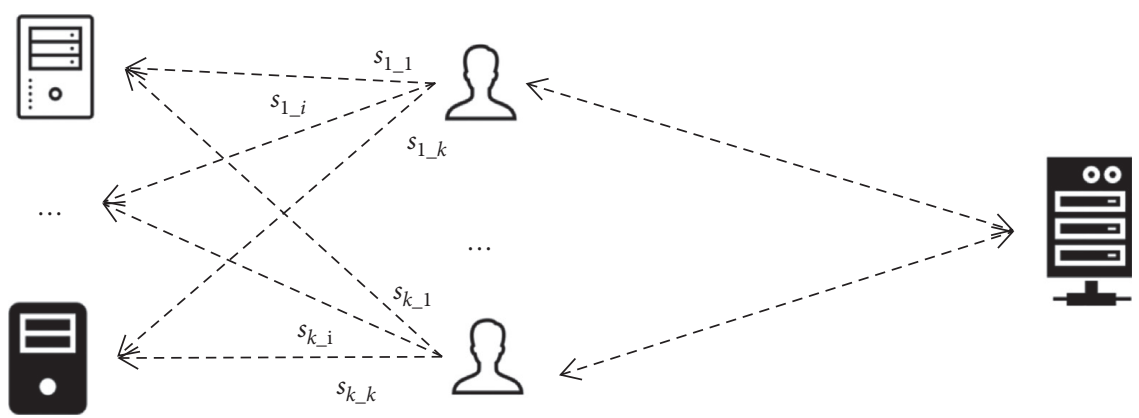

FIgURE 11: Architecture of our proposed scheme.

TABle 5: Notations used in our scheme.

\begin{tabular}{lc}
\hline Notations & Description \\
\hline$U_{X}, S_{j}, R C$ & $X^{\text {th }}$ user $/ j^{\text {th }}$ server, registration centre \\
$B_{X}$ & Biometrics of $U_{X}$ \\
$q_{R C}$ & Master key of $R C$ \\
$A S I D_{j}$ & $S_{j}^{\text {'s master key }}$ \\
$s_{j_{X}}$ & $U_{X}$ 's authentication key with $S_{j}$ \\
$S K$ & Session key between $U_{X}$ and $S_{j}$ \\
$H_{0}()$. & Cryptographic one-way hash function \\
Gen/Rep & Generation/reproduction algorithm \\
$\oplus, \|, T_{n}$ & XOR, concatenation, Chebyshev operation \\
\hline
\end{tabular}

3.6.3. User Registration Phase. $U_{X}$ provides biometrics $B_{X}$ and $U I D_{X}$, using Gen $\left(B_{X}\right)$ to generate $\left\langle R_{X}, P_{X}>\right.$. Then, $U_{X}$ sends $\left\{U I D_{X}, H_{0}\left(R_{X} \| U I D_{X}\right)\right\}$ to $R C$ through a secure channel. Once receiving the messages, $R C$ computes all submaster keys for all service providers. $R C$ chooses $r_{X}$ and then computes $s_{j_{X}}=T_{r_{X}}\left(U I D_{X} \| H_{0}\left(R_{X} \| U I D_{X}\right)\right) \bmod$ $p+T_{A S I D_{i}}\left(H_{0}\left(r_{j}+r_{X}+U I D_{X}\right)\right) \bmod p$ and $R P W_{X}=H_{0}\left(H_{0}\right.$ $\left.\left(R_{X} \| U I D_{X}\right) \| r_{X}\right) . R C$ returns $\left\{s_{1_{X}}, s_{2_{X}}, \ldots, s_{m_{X}}, R P W_{X}\right.$, and $H_{0}\left(\right.$.),$\left.r_{X}\right\}$ to $U_{X}$ through a secure channel. Figure 13 shows the steps in this phase.

In this phase, $R C$ computes $s_{j_{X}}$, which is an authentication key between $U_{X}$ and $S_{j}(1 \leq j \leq m$, where $m$ is a number of $S_{j}$ ). Similar to [19], our scheme uses the fuzzy extractor to deal with the problem of output-sensitive due to inputs' perturbations. Additionally, $R C$ must notify $S_{j}$ about $U_{X}$ by sending pair $\left\langle r_{X}, U I D_{X}\right\rangle$ for the subsequent user's authentication.

3.6.4. Authentication Phase. When $U_{X}$ logins to $S_{j}, U_{X}$ provides the smart card with $U I D_{X}$ and $B_{X}^{\prime}$ at the terminal. Then, the smart card reproduces $R_{X}=\operatorname{Rep}\left(P_{X}, B_{X}^{\prime}\right)$ and checks if $R P W_{X}=H_{0}\left(H_{0}\left(R_{X} \| U I D_{X}\right) \| r_{X}\right)$; if this does not hold, the session is terminated; otherwise, the smart card chooses $r_{U}$ and computes $T_{A S I D_{j}}\left(H_{0}\left(r_{j}+r_{X}+U I D_{X}\right)\right) \bmod$ $p=s_{j_{X}}-T_{r_{X}}\left(U I D_{X} \| H_{0}\left(R_{X} \| U I D_{X}\right)\right) \bmod p, R_{U}=T_{r_{U}}$ $\left(T_{A S I D_{j}}\left(H_{0}\left(r_{j}+r_{X}+U I D_{X}\right)\right) \bmod p\right) \bmod p, R^{\prime}=R_{U}+T_{A S I D_{j}}$ $\left(H_{0}\left(r_{j}+r_{X}+U I D_{X}\right)\right) \bmod p, C I D=U I D_{X} \oplus H_{0}\left(R_{U}\right)$, and $M_{U}=H_{0}\left(R_{U}, T_{A S I D_{j}}\left(H_{0}\left(r_{j}+r_{X}+U I D_{X}\right)\right) \bmod p\right)$. Then, the smart card sends $\left\{C I D, R^{\prime}, M_{U}, r_{X}\right\}$ to $S_{j}$. On receiving the message, $S_{j}$ computes $T_{A S I D_{j}}\left(H_{0}\left(r_{j}+r_{X}+U I D_{X}\right)\right) \bmod p$, $R_{U}^{\prime}=R^{\prime}-T_{A S I D_{i}} \quad\left(\begin{array}{ll}H_{0} & \left.\left(r_{j}+r_{X}+U I D_{X}\right)\right) \\ \bmod & p \text {, and }\end{array}\right.$ $U I D_{X}=C I D \oplus H_{0}\left(R_{U}^{\prime}\right)$ and checks $U I D_{X}$; then, $S_{j}$ checks if
$M_{U}=H_{0}\left(R_{U}^{\prime}, T_{A S I D_{j}}\left(H_{0}\left(r_{j}+r_{X}+U I D_{X}\right)\right) \bmod p\right)$, and if this does not hold, $S_{j}$ terminates the session; otherwise, $S_{j}$ chooses $r_{S}$ and computes $R_{S}=T_{r_{S}}\left(T_{A S I D_{j}}\left(H_{0}\left(r_{j}+r_{X}+U I D_{X}\right)\right) \bmod \right.$ p) $\bmod p, S^{\prime}=R_{S}+R_{U}^{\prime}, S K=H_{0}\left(T_{r_{S}}\left(R_{U}^{\prime}\right) \bmod p\right)$, and $M_{S}=H_{0}\left(R_{S}, T_{A S I D_{j}}\left(H_{0}\left(r_{j}+r_{X}+U I D_{X}\right)\right) \bmod p\right) . S_{j}$ sends $\left\{M_{S}, S^{\prime}\right\}$ to $U_{X}$. On receiving the message, $U_{X}$ computes $R_{S}^{\prime}=S^{\prime}-R_{U}$ and $S K=H_{0}\left(T_{r_{U}}\left(R_{S}^{\prime}\right) \bmod p\right)$ and checks if $M_{S}=H_{0}\left(R_{S}^{\prime}, T_{A S I D_{j}}\left(H_{0}\left(r_{j}+r_{X}+U I D_{X}\right)\right) \bmod p\right)$; if this does not hold, $U_{X}$ terminates the session; otherwise, $U_{X}$ believes $S_{j}$ is valid and sends $M_{U S}=H_{0}\left(R_{S}^{\prime}, T_{r_{U}}\left(R_{S}^{\prime}\right) \bmod p\right)$ to $S_{j}$. On receiving the message, $S_{j}$ checks if $M_{U S}=H_{0}\left(R_{S}, T_{r_{S}}\left(R_{U}^{\prime}\right)\right.$ $\bmod p$ ); if this does not hold, $S_{j}$ terminates the session; otherwise, $S_{j}$ believes $U_{X}$ is valid. Figure 14 shows the steps in this phase.

3.6.5. Password-Update Phase. When $U_{X}$ changes $B_{X}, U_{X}$ provides his/her smart card with $U I D_{X}$ and similar $B_{X}^{\prime}$ at the terminal. Then, the smart card checks if $R P W_{X}=H_{0}\left(H_{0}\right.$ $\left.\left(R_{X} \| U I D_{X}\right) \| r_{X}\right)$, where $R_{X}=\operatorname{Rep}\left(P_{X}, B_{X}^{\prime}\right)$. If this does not hold, the smart card terminates the session; otherwise, $U_{X}$ inputs $B_{\text {new }}$ and computes $R P W_{\text {new }}=H_{0} \quad\left(H_{0} \quad\left(R_{\text {new }} \|\right.\right.$ $\left.\left.U I D_{X}\right) \| r_{X}\right)$, where $<R_{\text {new }}, P_{\text {new }}>=\operatorname{Gen}\left(B_{\text {new }}\right)$, Then, the smart card updates $R P W_{\mathrm{X}}=R P W_{\text {new }}$ and $P_{X}=P_{\text {new }}$. Finally, the smart card updates all authentication keys $s_{j_{X}}=s_{j_{X}}-T_{r_{X}}$ $\left(U I D_{X} \| H_{0}\left(R_{X} \| U I D_{X}\right)\right)+T_{r_{X}}\left(U I D_{X} \| H_{0}\left(R_{\text {new }} \| U I D_{X}\right)\right)^{X}$, $\forall j$.

\section{Security and Efficiency Analyses}

In this section, we analyse our scheme on security and efficiency aspects.

4.1. Correctness Analysis. Similar to previous schemes, we also prove our scheme's correctness using BAN-logic rules [28] and goals proposed in [29]. For simplicity, we let $\otimes$ denote the combination using Chebyshev operation. Table 6 shows some assumptions our scheme must satisfy.

These assumptions stand for initial beliefs of the user and server, for example, $A_{1}$ implies that users can share their identities with the server with the registration phase. Next, we will normalize all messages exchanged between the user and server.

(i) From the message $\{C I D\}$, we have $<U_{X} \stackrel{U I D_{X}}{\longleftrightarrow} S_{j}$, $U_{X} \stackrel{s_{j_{X}}}{\longleftrightarrow} S_{j}, r_{U} \otimes s_{j_{X}}>$ 
$S_{j}$ $R C\left(q_{\mathrm{RC}}\right)$

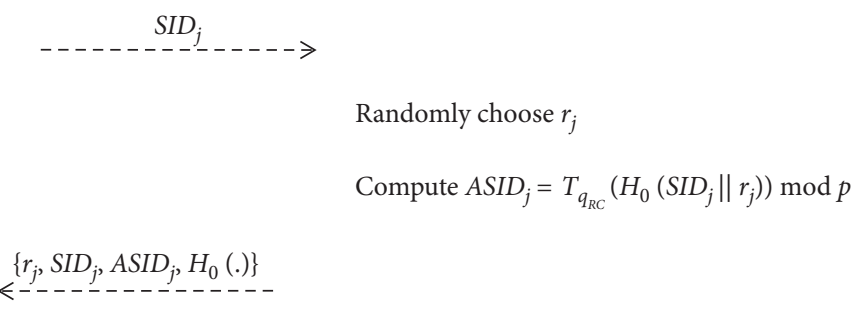

Keep secret data

Figure 12: Proposed scheme's server registration phase.

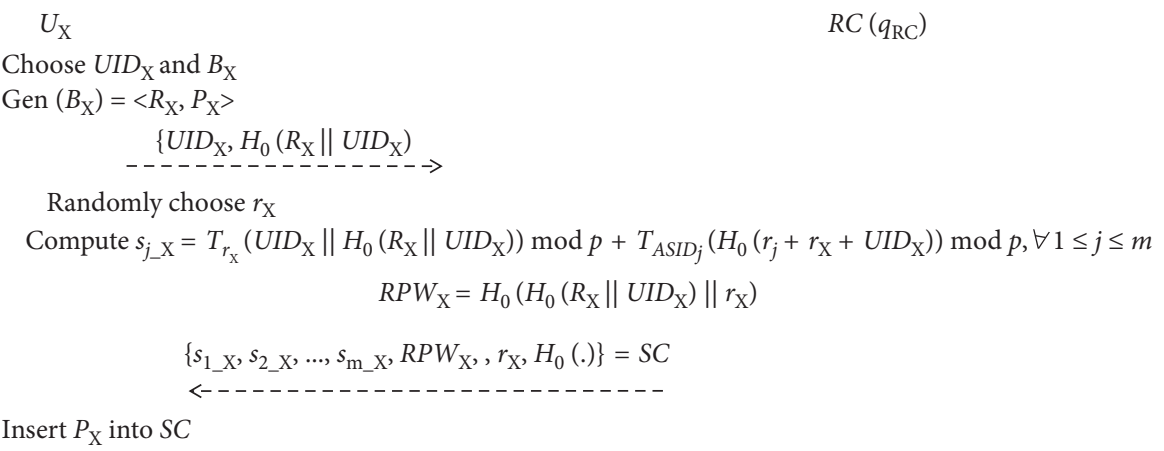

FIGURE 13: Proposed scheme's user registration phase.

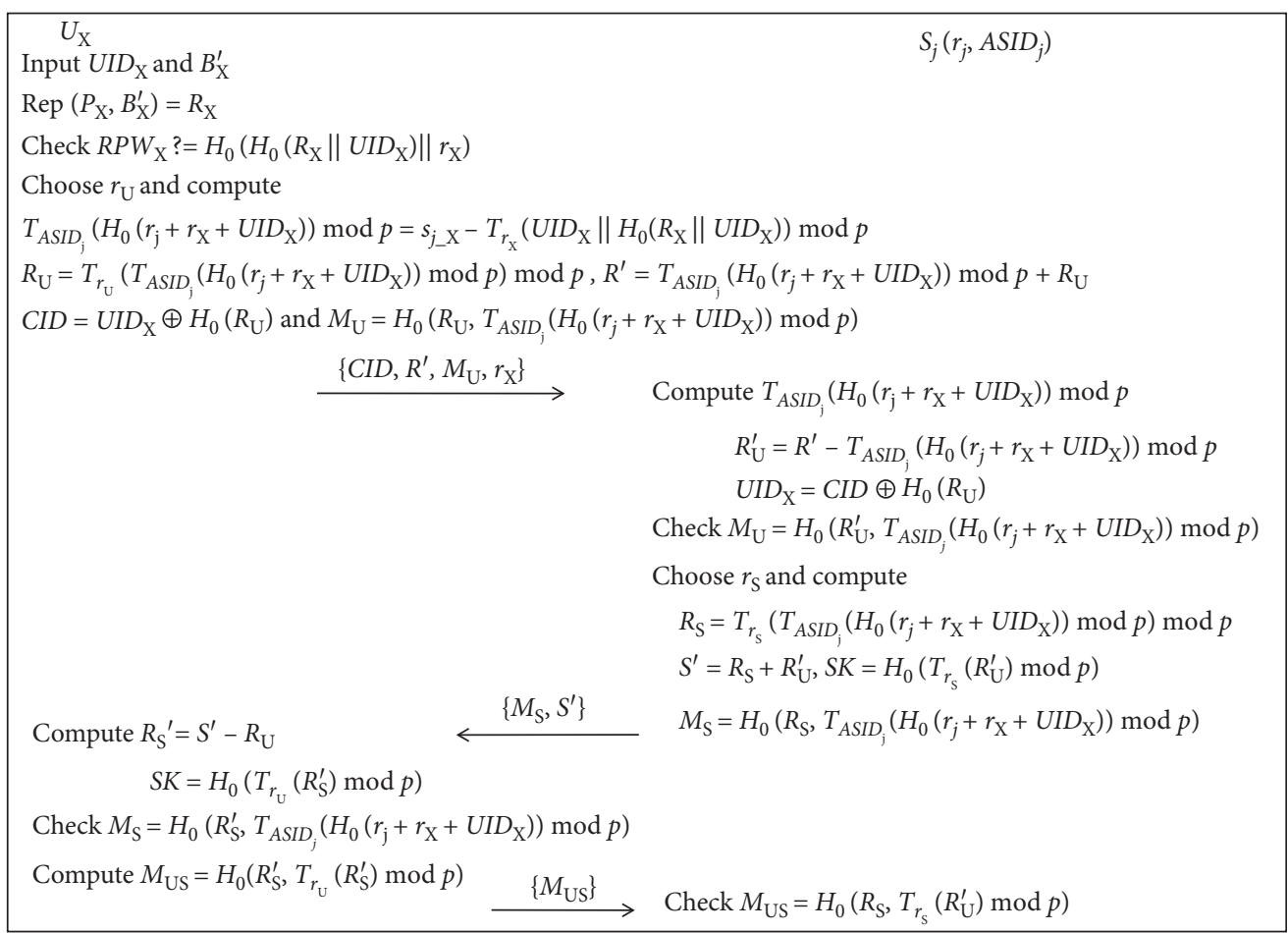

Figure 14: Proposed scheme's authentication phase.

(ii) From ${ }_{j_{j_{X}}}$ the message $\left\{M_{U}\right\}$, we have $<r_{U} \otimes s_{j_{X}}$, $U_{X} \stackrel{j_{X}}{\longleftrightarrow} S_{j}>$

(iii) From the third messages $\left\{M_{S}\right\}$, we have $<r_{S} \otimes s_{j_{X}}$, $U_{X} \stackrel{j_{X}}{\longleftrightarrow} S_{j}>$ (iv) From the fourth f $_{S K}$ message $\left\{M_{U S}\right\}$, we have $<U_{X} \stackrel{s_{j_{X}}}{\longleftrightarrow} S_{j}, U_{X} \stackrel{S K}{\longleftrightarrow} S_{j}>$

The normalization helps to clearly show information exchanged between $U_{X}$ and $S_{j}$, for example, CID containing 
TABLE 6: The assumptions in BAN-logic.

Assumption

$A_{1}: U_{X} \mid \equiv\left(U_{X} \stackrel{U I D_{X}}{\longleftrightarrow} S_{j}\right)-U_{X}$ believes $U_{X}$ can share $U I D_{X}$ with $S_{j}$

$A_{2}: U_{X} \mid \equiv\left(U_{X} \stackrel{s_{j_{X}}}{\longleftrightarrow} S_{j}\right)-U_{X}$ believes $U_{X}$ can share $s_{j_{X}}$ with $S_{j}$

$A_{3}: U_{X} \mid \equiv\left(S_{j} \Rightarrow\left(U_{X} \stackrel{S K}{\longleftrightarrow} S_{j}\right)\right)-U_{X}$ believes $S_{j}$ controls the sharing of the session key between $U_{X}$ and $S_{j}$

$A_{4}: S_{j} \mid \equiv\left(U_{X} \Rightarrow\left(U_{X} \stackrel{U I D_{X}}{\longleftrightarrow} S_{j}\right)\right)-S_{j}$ believes $U_{X}$ controls the sharing of $U I D_{X}$ between $U_{X}$ and $S_{j}$

$A_{5}: S_{j} \mid \equiv\left(U_{X} \Rightarrow\left(U_{X} \stackrel{S K}{\longleftrightarrow} S_{j}\right)\right)-S_{j}$ believes $U_{X}$ controls the sharing of the session key between $U_{X}$ and $S_{j}$

$A_{6}: S_{j} \mid \equiv\left(S_{j} \stackrel{s_{j_{X}}}{\longleftrightarrow} U_{X}\right)-S_{j}$ believes $S_{j}$ can share $s_{j_{X}}$ with $U_{X}$

$A_{7}: U_{X} \mid \equiv \#\left(r_{S} \otimes s_{j_{X}}\right)-U_{X}$ believes challenge messages from $S_{j}$ are fresh

$A_{8}: S_{j} \mid \equiv \#\left(r_{U} \otimes s_{j_{X}}\right)-S_{j}$ believes challenge messages from $U_{X}$ are fresh

identity, challenge information $r_{U} \otimes s_{j_{X}}$, and long-term key $s_{j_{X}}$. Next, we demonstrate how our scheme satisfies seven lemmas reorganized from [29].

Lemma 1. If $S_{j}$ believes the authentication key (the long-term key) is successfully shared with $U_{X}$ and $U_{X}$ 's messages encrypted with this key are fresh, $S_{j}$ will believe that $U_{X}$ believes $U_{X}$ 's $U I D_{X}$ is successfully shared with $S_{j}$ :

$$
\frac{S_{j}\left|\equiv\left(S_{j} \stackrel{s_{j_{X}}}{\longleftrightarrow} U_{X}\right), S_{j}\right| \equiv \#\left(r_{U} \otimes s_{j_{X}}\right)}{S_{j}\left|\equiv\left(U_{X} \equiv\left(U_{X} \stackrel{U I D_{X}}{\longleftrightarrow} S_{j}\right)\right)\right|} .
$$

Proof. With $A_{6}$ and $C I D$, we apply the message-meaning rule to

have

$S_{j}\left|\equiv\left(S_{j} \stackrel{s_{j_{X}}}{\longleftrightarrow} U_{X}\right), S_{j} \triangleleft \mathrm{CID} / S_{j}\right| \equiv\left(_{U X} \mid \sim \mathrm{CID}\right)$. With $A_{8}$, we apply the freshness rule to have $S_{j}\left|\equiv \#\left(r_{U} \otimes s_{j_{X}}\right) / S_{j}\right| \equiv \#$ CID. Next, we apply the nonceverification rule to have $S_{j}\left|\equiv U_{X}\right| \sim C I D, S_{j} \mid \equiv \#$ $\mathrm{CID} / S_{j}\left|\equiv U_{X}\right| \equiv$ CID. Finally, we apply the believe rule to have $S_{j}\left|\equiv U_{X}\right| \equiv \mathrm{CID} / S_{j}\left|\equiv U_{X}\right| \equiv U_{X} \stackrel{U I D_{X}}{\longleftrightarrow} S_{j}$. So, with $A_{6}$ and $A_{8}$, we successfully demonstrate how our scheme satisfies Lemma 1.

Lemma 2. If $S_{j}$ believes $U_{X}$ also believes $U_{X}$ 's $U I D_{X}$ is successfully shared with each other and $U_{X}$ totally controls this $U I D_{X}$ 's sharing, $S_{j}$ also believes $U_{X}$ 's $U I D_{X}$ is successfully shared with each other:

$$
\frac{S_{j}\left|\equiv\left(U_{X} \mid \equiv\left(U_{X} \stackrel{U I D_{X}}{\longleftrightarrow} S_{j}\right)\right), S_{j}\right| \equiv\left(U_{X} \Longrightarrow\left(U_{X} \stackrel{U I D_{X}}{\longleftrightarrow} S_{j}\right)\right)}{S_{j} \mid \equiv U_{X} \stackrel{U I D_{X}}{\longleftrightarrow} S_{j}} .
$$

Proof. With Lemma 1 and $A_{4}$, we apply the jurisdiction rule to have $S_{j}\left|\equiv U_{X}\right| \equiv\left(U_{X} \stackrel{U I D_{X}}{\longleftrightarrow} S_{j}\right), S_{j} \mid \equiv U_{X} \Rightarrow\left(U_{X}\right.$ $\left.\stackrel{U I D_{X}}{\longleftrightarrow} S_{j}\right) / S_{j} \mid \equiv U_{X} \stackrel{U I D_{X}}{\longleftrightarrow} S_{j}$. So, with Lemma 1 and $A_{4}$, we prove how our scheme satisfies Lemma 2.
Lemma 3. If $U_{X}$ believes $s_{j_{X}}$ is successfully shared with $S_{j}$ and $S_{j}$ 's messages encrypted with $s_{j_{X}}$ are fresh, $U_{X}$ will believe $S_{j}$ also believes $U_{X}$ 's $U I D_{X}$ is successfully shared with each other.

Proof. With $A_{2}$ and $M_{S}$, we apply the jurisdiction rule to have $U_{X}\left|\equiv\left(U_{X} \stackrel{s_{j_{X}}}{\longleftrightarrow} S_{j}\right), U_{X} \triangleleft M_{S} / U_{X}\right| \equiv S_{j} \mid \sim M_{S}$. Then, with $A_{7}$, we apply the freshness rule to have $U_{X}\left|\equiv \#\left(r_{S} \otimes s_{j_{X}}\right), U_{X}\right| \equiv S_{j}\left|\sim M_{S} / U_{X}\right| \equiv \# M_{S}$. So, combining two results with the nonce-verification rule, we have $U_{X}\left|\equiv S_{j}\right| \sim M_{S}, U_{X}\left|\equiv \# M_{S} / U_{X}\right| \equiv S_{j} \mid \equiv M_{S}$. Finally, we apply the believe rule to have $U_{X}\left|\equiv S_{j}\right| \equiv M_{S} / U_{X} \mid \equiv$ $S_{j} \mid \equiv U_{X} \stackrel{U I D_{X}}{\longleftrightarrow} S_{j}$. So, with $A_{2}$ and $A_{7}$, we successfully prove how our scheme satisfies Lemma 3. In short, with three lemmas, we can say that both $S_{j}$ and $U_{X}$ believe and successfully share their identities with each other. Next, we need to prove the similar thing for the session key.

Lemma 4. If $U_{X}$ believes that $s_{j_{X}}$ is successfully shared with $S_{j}$ and $S_{j}$ 's messages encrypted with $s_{j_{X}}$ are fresh, $U_{X}$ will believe $S_{j}$ also believes the session key $S K$ is successfully shared with each other:

$$
\frac{U_{X}\left|\equiv\left(U_{X} \stackrel{s_{j_{X}}}{\longleftrightarrow} S_{j}\right), U_{X}\right| \equiv \#\left(r_{S} \otimes s_{j_{X}}\right)}{U_{X}\left|\equiv S_{j}\right| \equiv S_{j} \stackrel{S K}{\longleftrightarrow} U_{X}} .
$$

Proof. With $M_{U S}$ and $A_{2}$, we apply the message-meaning rule to have $U_{X}\left|\equiv\left(U_{X} \stackrel{s_{j_{X}}}{\longleftrightarrow} S_{j}\right), U_{X} \triangleleft M_{U S} / U_{X}\right| \equiv S_{j} \mid \sim M_{U S}$. With $A_{7}$ and $M_{U S}$, we apply the freshness rule to have $U_{X}\left|\equiv \#\left(r_{S} \otimes s_{j_{X}}\right), U_{X} \triangleleft M_{U S} / U_{X}\right| \equiv \# M_{U S}$. Next, we use the believe rule to have $U_{X}\left|\equiv S_{j}\right| \sim M_{U S}, U_{X} \mid \equiv$ $\# M_{U S} / U_{X}\left|\equiv S_{j}\right| \equiv M_{U S}$. Again, we apply the believe rule to have $U_{X}\left|\equiv S_{j}\right| \equiv M_{U S} / U_{X}\left|\equiv S_{j}\right| \equiv S_{j} \stackrel{S K}{\longleftrightarrow} U_{X}$. So, with $A_{2}$ and $A_{7}$, we successfully prove how our scheme satisfies Lemma 4.

Lemma 5. If $U_{X}$ believes $S_{j}$ totally controls $S K$ 's sharing and $S_{j}$ also believes $S K$ is successfully shared with $U_{X}, U_{X}$ will believe SK's sharing: 


$$
\frac{U_{X}\left|\equiv S_{j}\left(U_{X} \stackrel{S K}{\longleftrightarrow} S_{j}\right), U_{X}\right| \equiv S_{j} \mid \equiv\left(S_{j} \stackrel{S K}{\longleftrightarrow} U_{X}\right)}{U_{X} \mid \equiv U_{X} \stackrel{S K}{\longleftrightarrow} S_{j}} .
$$

Proof. With $A_{3}$ and Lemma 4, we apply the jurisdiction rule to have $\quad U_{X}\left|\equiv S_{j}\left(U_{X} \stackrel{S K}{\longleftrightarrow} S_{j}\right), U_{X}\right| \equiv S_{j} \mid \equiv$ $\left(S_{j} \stackrel{S K}{\longleftrightarrow} U_{X}\right) / U_{X} \mid \equiv U_{X} \stackrel{S K}{\longleftrightarrow} S_{j}$. So, with $A_{3}$ and Lemma 4 , we successfully prove how our scheme satisfies Lemma 5.

Lemma 6. If $S_{j}$ believes $s_{j_{X}}$ is successfully shared with $U_{X}$ and the $U_{X}$ 's messages encrypted with $s_{j_{X}}$ are fresh, $S_{j}$ will believe $U_{X}$ also believes SK's sharing:

$$
\frac{U_{X}\left|\equiv\left(S_{j} \stackrel{s_{j_{X}}}{\longleftrightarrow} U_{X}\right), S_{j}\right| \equiv \#\left(r_{U} \otimes s_{j_{X}}\right)}{S_{j}\left|\equiv U_{X}\right| \equiv U_{X} \stackrel{S K}{\longleftrightarrow} S_{j}} .
$$

Proof. With $A_{6}$ and $M_{U S}$, we apply the message-meaning rule to have $S_{j}\left|\equiv\left(S_{j} \stackrel{s_{j_{X}}}{\longleftrightarrow} U_{X}\right), S_{j} \triangleleft M_{U S}\right| S_{j}\left|\equiv U_{X}\right| \sim M_{U S}$. With $A_{8}$ and $M_{U S}$, we apply the freshness rule to have $S_{j}\left|\equiv \#\left(r_{U} \otimes s_{j_{X}}\right), S_{j} \triangleleft M_{U S} / S_{j}\right| \equiv \# M_{U S}$. With two results and the nonce-verification rule, we have $S_{j}\left|\equiv U_{X}\right| \sim M_{U S}, S_{j}\left|\equiv \# M_{U S}\right| S_{j}\left|\equiv U_{X}\right| \equiv M_{U S}$. Finally, with $A_{6}$ and the believe rule, we have $S_{j} \mid \equiv$ $\left(S_{j} \stackrel{s_{j_{X}}}{\longleftrightarrow} U_{X}\right), S_{j}\left|\equiv U_{X}\right| \equiv M_{U S} / S_{j}\left|\equiv U_{X}\right| \equiv U_{X} \stackrel{S K}{\longleftrightarrow} S_{j}$. So, with $A_{6}$ and $A_{8}$, we successfully prove how our scheme satisfies Lemma 6.

Lemma 7. If $S_{j}$ believes $U_{X}$ totally controls $S K$ 's sharing, $S_{j}$ believes $S K$ is successfully shared with $U_{X}$ :

$$
\frac{U_{X}\left|\equiv S_{j}\left(U_{X} \stackrel{S K}{\longleftrightarrow} S_{j}\right), U_{X}\right| \equiv S_{j} \mid \equiv\left(S_{j} \stackrel{S K}{\longleftrightarrow} U_{X}\right)}{U_{X} \mid \equiv U_{X} \stackrel{S K}{\longleftrightarrow} S_{j}} .
$$

Proof. With $S_{j}\left|\equiv U_{X}\right| \equiv M_{U S}$ and $A_{5}$, we apply the messagemeaning rule to have $S_{j}\left|\equiv U_{X}\left(U_{X} \stackrel{S K}{\longleftrightarrow} S_{j}\right), S_{j}\right| \equiv U_{X} \mid$ $\equiv M_{U S} / S_{j} \mid \equiv M_{U S}$. Finally, we apply the believe rule to have $S_{j}\left|\equiv M_{U S} / S_{j}\right| \equiv S_{j} \stackrel{S K}{\longleftrightarrow} U_{X}$. So, with $A_{5}$, we completely prove how our scheme satisfies Lemma 7. Finally, we can say that both $S_{j}$ and $U_{X}$ believe the common $S K$ in our scheme.

4.2. Authenticated Key Exchange Security Analysis (AKESecurity). The adversarial model is presented in this section, and some definitions can be found in $[17,30]$. At first, we use some notations standing for the instances of our scheme's participants:

(i) $R C^{k}$ : the $k^{\text {th }}$ registration centre holding secret $q_{R C}$

(ii) $S_{j}^{i}$ : the $i^{\text {th }}$ server holding $\left\{r_{j}, A S I D_{j}\right\}$

(iii) $U_{X}^{i}$ : the $i^{\text {th }}$ user holding the smart card and $\left\{U I D_{X}\right.$, $\left.B_{X}\right\}$ (iv) $O_{\text {Hash }}$ : the hash oracle can be viewed as random functions

(v) $\operatorname{sid}_{U_{X}^{i}}^{S_{j}^{i}}$ and $\operatorname{sid}_{S_{j}^{i}}^{U_{X}^{i}}$ : the all messages exchanged between $U_{X}^{i}$ and $S_{j}^{i}$

(vi) $\operatorname{pid}_{U_{X}^{i}}=S_{j}^{i}$ and $p i d_{S_{j}^{i}}=U_{X}^{i}$ : the partner identity of $U_{X}^{i}$ is $S_{j}^{i}$ and vice versa

Next, there are some security properties:

(i) $S_{j}^{i}$ and $U_{X}^{i}$ are accepted if they can compute the valid session key and receive expected messages

(ii) $S_{j}^{i}$ and $U_{X}^{i}$ are partnered if (1) both of them are accepted, (2) pid $U_{U_{X}^{i}}=S_{j}^{i} \quad\left(p_{i d_{S_{j}^{i}}}=U_{X}^{i}\right)$, and (3) $\operatorname{sid}_{U_{X}^{i}}^{j}=\operatorname{sid}_{S_{i}^{i}}^{U_{X}^{i}}$

(iii) $U_{X}^{i}$ are fresh if (1) they are partnered, (2) no secret information of $U_{X}^{i}$ is leaked before it is accepted, (3) no $U_{X}^{i}$ 's session key is leaked before it is accepted

Next, there are some adversary's capabilities. Let $A$ be probabilistic polynomial time adversary attacking authentication scheme in AKE-security:

(i) Execution query helps $A$ to execute passive attacks against our scheme. We let $q_{E}$ be the sum of the number of execute queries and $\operatorname{sid}_{U^{i}}^{{ }^{i}} \leftarrow$ Execute $\left(U_{X}^{i}, S_{j}^{i}\right)$ be the symbol standing for the output of this query.

(ii) Send query helps $A$ to actively interact with $U_{X}^{i}$ or $S_{j}^{i}$. We let $q_{S}$ be the sum of the number of send queries and $m_{\text {out }} \leftarrow$ Send $\left(O^{i}, m_{\text {in }}\right)$ be the symbol standing for the output and input of this query, where $\mathrm{O}^{\mathrm{i}}$ is $U_{X}^{i}$ or $S_{j}^{i}$.

(iii) Reveal query helps $A$ to know the session key ( $s k$ ) of $U_{X}^{i}$ and $S_{j}^{i}$ in another session. We let $q_{R}$ be the sum of the number of reveal queries and $s k^{i} \leftarrow$ Reveal $\left(\mathrm{O}^{\mathrm{i}}\right)$ be the symbol standing for the output of this query, where $\mathrm{O}^{\mathrm{i}}$ is $U_{X}^{i}$ or $S_{j}^{i}$. Note that to perform this query, $\mathrm{O}^{\mathrm{i}}$ must be fresh.

(iv) Corrupt query helps $A$ to know secret information of $U_{X}^{i}$. We let $q_{C}$ be the sum of the number of corrupt queries and $\left\{U I D_{X}, B_{X}\right\} \leftarrow$ Corrupt $\left(U_{X}^{i}, 1\right)$ and $\{$ smart card $\} \leftarrow$ Corrupt $\left(U_{X}^{i}, 0\right)$ be the symbol standing for the output of this query. Note that $A$ only knows the smart card or $\left\{U I D_{X}, B_{X}\right\}$.

(v) Hash query helps $A$ to query a value $m$ and receive corresponding $r$. If $m$ is not queried before, $O_{\text {Hash }}$ returns the random number $r$ to the adversary. Otherwise, it returns the previously generated result. We let $q_{H}$ be the sum of the number of hash queries and $r \leftarrow$ Hash $\left(O_{\text {Hash }}, m\right)$ be the symbol standing for the output of this query.

Theorem 1. Our scheme $P$ is AKE-security against $A$ within a time $t_{A}$ if $A$ cannot guess the correct session key of another fresh $O^{i}$. Formally, let $A d v_{P}^{A K E}\left(A, t_{A}\right)$ be the $A$ 's chance of breaking $P$ in $A K E$-security within in reasonable time $t_{A}$, and we need $A d v_{P}^{A K E}\left(A, t_{A}\right)$ is negligible. In summary, we need $A d v_{P}^{A K E}\left(A, t_{A}\right) \leq \varepsilon(*)$. 
4.3. The Definition of Experiments. We set $\operatorname{Exp}_{T}^{C M D H P}(B)$ be experiment, where the adversary $B$ wins if it successfully breaks CMDHP-security of the Chebyshev polynomial $T^{\circ} \mathrm{s}$ problem within a time $t_{\mathrm{B}}$, and let $A d v_{T}^{C M D H P}(B)=\mathrm{Pr}$ $\left[\operatorname{Exp}_{T}^{C M D H P}(B)=1\right]$ be the $B$ 's winning chance. Note that we give $O_{\text {Hash }}$ to $B$.

\section{Experiment 1}

$\operatorname{Exp}_{T}^{C M D H P}(B)$

$$
\begin{aligned}
& \{s, r\} \stackrel{\$}{\longleftarrow}\{0,1\}^{*}, X \stackrel{\$}{\longleftarrow}[0, p-1] \\
& M \longleftarrow T_{r}(X) \bmod p \\
& N \longleftarrow T_{s}(X) \bmod p \\
& K \longleftarrow T_{r}\left(T_{s}(X)\right) \bmod p \\
& Z \longleftarrow B_{\text {Hash }}^{O}\left(M, N, \text { Hash }\left(O_{\mathrm{Hash}}, K\right)\right) \\
& \text { if Hash }\left(O_{\mathrm{Hash}}, Z\right)=\text { Hash }\left(O_{\mathrm{Hash}}, K\right) \text { then return } 1 \\
& \text { else return } 0
\end{aligned}
$$

Also, we set $\operatorname{Exp}_{P}^{A K E}(A)$ be experiment, where the adversary $A$ wins if it successfully breaks AKE-security of our $P$ within a time $t_{A}$, and let $A d v_{P}^{A K E}(A)=\operatorname{Pr}\left[\operatorname{Exp} p_{P}^{A K E}\right.$ $(A)=1$ ] be the $A$ 's winning chance. Note that we also give $O_{\text {Hash }}$ to $A$.

\section{Experiment 2}

$\operatorname{Exp}_{P}^{A K E}(A)$.

$$
\begin{aligned}
& b \stackrel{\$}{\longleftarrow}\{0,1\}, L_{\mathrm{U}} \longleftarrow\left\{U_{1}, \ldots, U_{n}\right\}, L_{\mathrm{S}} \longleftarrow\left\{S_{1}, \ldots, S_{n}\right\} \\
& U_{i} \stackrel{\$}{\longleftarrow} L_{U}, S_{j} \stackrel{\$}{\longleftarrow} L_{S} \\
& \operatorname{sid}_{U_{i}}^{S_{j}} \longleftarrow \text { Execute }\left(U_{i}, S_{j}\right) \\
& \text { if } b=0 \text { then } s k \stackrel{\$}{\longleftarrow}\{0,1\}^{*} \\
& \text { else } s k \longleftarrow \text { Reveal }\left(U_{i}\right) \\
& \left\{b^{\prime}, Z\right\} \longleftarrow A^{O_{\text {Hash }}}\left(L_{U}, L_{S}, s k\right) \\
& \text { if } b^{\prime}=b \text { then return } 1 \\
& \text { else return } 0
\end{aligned}
$$
$Z)=s k$.

Note that if $b=b^{\prime}=1$, then we must have Hash $\left(O_{\text {Hash}}\right.$,

The proof of Theorem 1 .

Now we assume that $B$ wants to win in $B$ 's experiment, and it runs $A$ as the procedure. Also, $A$ wants to win in $A$ 's experiment and $B$ must simulate the $A$ 's environment as the following algorithm Let $l$ be the security length, for example, the size of the prime $p$ and hash function's output. If $A$ correctly guesses $b^{\prime}$, then we must consider some following cases (Algorithm 1):

(i) $A$ issues $q_{H}$ queries to $O_{\text {Hash }}$, and $A$ has successful probability $\approx q_{H}^{2} / 2^{l}$ due to the birthday paradox.

(ii) $A$ chooses $q_{E}$ pairs to execute and have all messages exchanged between them. Furthermore, $A$ issues $q_{C}$ queries to some users to get the smart card or $\{U I D, B\}$. So, $A$ 's successful probability of correctly guessing random values $r$ or $s$ is $\approx q_{E} \times q_{C} / p$.

(iii) If $\mathrm{A}$ issues $q_{S}^{\prime}$ queries to oracles simulated by $\mathrm{B}$, there will be at least one Send query that helps $A$ compute the session key. So, we have $A d v_{T}^{C M D H P}$ (B) $\geq$ $A d v_{P}^{A K E}(A) / q_{S}^{\prime}$. When $A$ issues the remaining $q_{S}-$ $q_{S}^{\prime}$ queries to normal $O^{i}, A^{\prime}$ 's successful probability of correctly guessing is $\approx q_{S}-q_{S}^{\prime} / p$.

Finally, we have $A d v_{P}^{A K E} \quad(A) \leq q_{H}^{2} / 2^{l}+q_{E} \times q_{C}$ l $p+A d v_{T}^{C M D H P}(B) \times q_{S}^{\prime}+q_{S}-q_{S}^{\prime} / p$. Clearly, the right-hand side of this inequality is negligible, so we complete the proof.

4.4. Security Analysis. In this section, we will analyse our scheme in some popular kinds of attacks. The comparison of the security feature with previous works is shown in Table 7. Before coming into this section, we want to discuss the privileged insider attacks. In our scheme, we use biometrics rather than the password. Therefore, the privileged insider attacker cannot find it and exploit into different servers. Furthermore, all servers in our scheme use different long-term keys provided from RC, so the attacker cannot use the users' information in another server to exploit in others. It can be said that it is hard to launch a privileged insider attack in our scheme.

4.5. Password-Guessing Attack. In this kind of attack, the adversary can guess the user's password by exploiting leaking information from the smart card. The adversary will have $R P W_{X}=H_{0} \quad\left(H_{0} \quad\left(R_{X} \| U I D_{X}\right) \| r_{X}\right)$. Because $R_{U}$ is produced by the user's biometrics, it is hard to guess. Clearly, the adversary cannot use the dictionary method to find biometrics and our scheme can resist this kind of attack.

4.6. User's Anonymity. In this kind of attack, the adversary eavesdrops $\left\{r_{X}, C I D, M_{U}, R^{\prime}\right\},\left\{S^{\prime}, M_{S}\right\}$, and $\left\{M_{U S}\right\}$ from $U_{X}$. All messages are different at each login session because we use random values. So, the adversary cannot trace who is online. In other words, our scheme resists this attack.

4.7. Two-Factor Attack. Although $U_{X}$ loses his/her smart card, the adversary cannot exploit because of needing $B_{X}$ to pass $R P W_{X}=H_{0}\left(H_{0}\left(R_{X} \| U I D_{X}\right) \| r_{X}\right)$. Even if $U_{X}$ 's biometrics is fake, the adversary cannot exploit all information in the login message because he/she does not other supporting values. Clearly, our scheme resists this attack.

4.8. Known Session-Specific Information Attack. When $r_{U}$ and $r_{S}$ are leaked, the adversary cannot compute the session key $(S K)$. He needs $T_{r_{U}}\left(T_{r_{S}}\left(T_{A S I D_{j}}\left(H_{0}\left(r_{j}+r_{X}+U I D_{X}\right)\right)\right.\right.$ $\bmod p) \bmod p) \bmod p$ to compute the $S K$. If he does not have the smart card's information, all important keys cannot be successfully computed. Clearly, our scheme can resist this kind of attack.

4.9. Session-Key Perfect Forward Secrecy. If all important keys are leaked, the adversary cannot compute previous transactions between $U_{X}$ and $S_{j}$. With $A S I D_{j}$ and all messages $U_{X}$ sent to $S_{j}$, the adversary extracts $R_{U}=R^{\prime}-T_{A S I D_{j}}\left(H_{0}\right.$ $\left.\left(r_{j}+r_{X}+U I D_{X}\right)\right) \bmod p$ and $R_{S}=S^{\prime}-R_{U}$. Finally, the adversary cannot compute $T_{r_{U}} \quad\left(T_{r_{S}} \quad\left(T_{A S I D_{j}} \quad\left(H_{0}\right.\right.\right.$ 


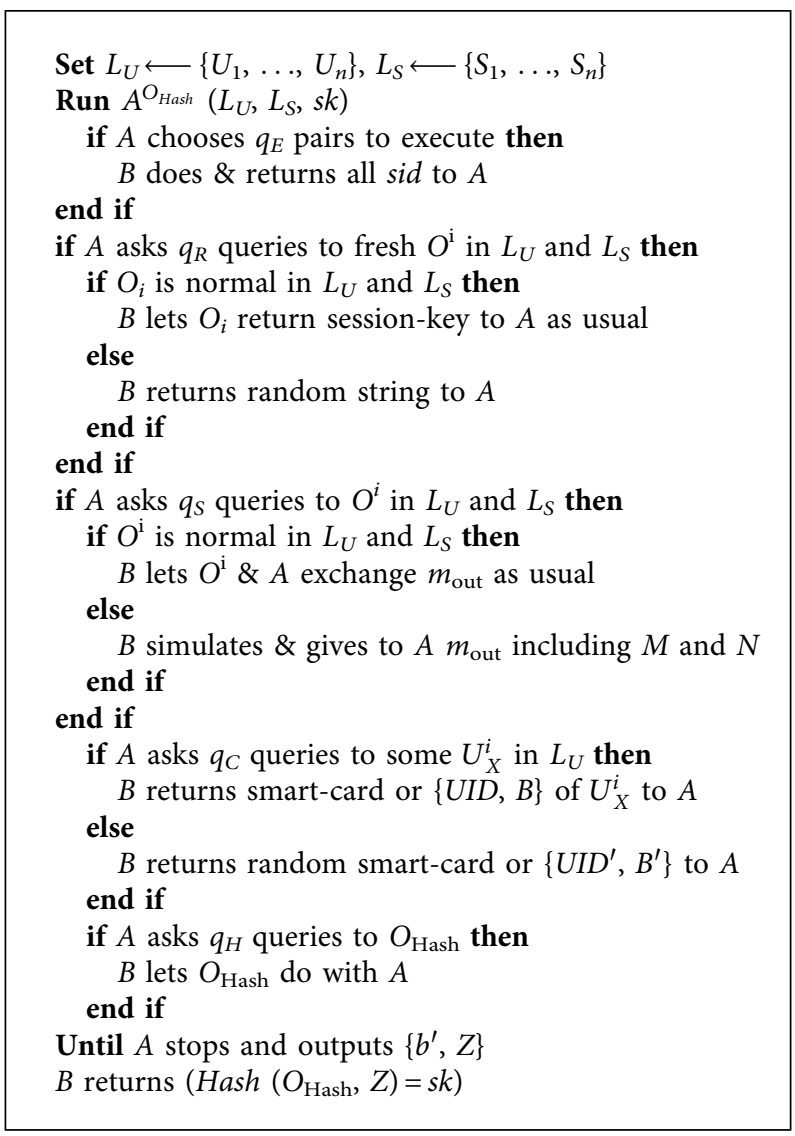

Algorithm 1: $B_{T}^{C M D H P}\left(M, N, s k \longleftarrow\right.$ Hash $\left.\left(O_{\text {Hash }}, K\right)\right)$.

TABle 7: The security feature comparison among the schemes.

\begin{tabular}{|c|c|c|c|c|c|c|}
\hline Schemes & Li [11] & $\mathrm{Qu}[12]$ & Amin [13] & Jangirala [18] & Han [19] & Ours \\
\hline Password-guessing & $x$ & $\checkmark$ & $\checkmark$ & $\checkmark$ & $\checkmark$ & $\sqrt{ }$ \\
\hline User's anonymity & $\checkmark$ & $\checkmark$ & $\checkmark$ & $\sqrt{ }$ & $\checkmark$ & $\checkmark$ \\
\hline Two-factor & $x$ & $\checkmark$ & $\checkmark$ & $x$ & $\checkmark$ & $\checkmark$ \\
\hline Known session-specific temporary information & $x$ & $\checkmark$ & $\checkmark$ & $x$ & $x$ & $\checkmark$ \\
\hline Session-key perfect forward & $x$ & $\checkmark$ & $x$ & $x$ & $\checkmark$ & $\checkmark$ \\
\hline User impersonation & $x$ & $x$ & $\checkmark$ & $x$ & $x$ & $\checkmark$ \\
\hline Server impersonation & $\checkmark$ & $\checkmark$ & $\checkmark$ & $\checkmark$ & $\checkmark$ & $\checkmark$ \\
\hline Man-in-the-middle & $\checkmark$ & $\overbrace{}^{1}$ & $\overbrace{}^{1}$ & $\mathrm{~s}^{1}$ & $\oiint^{1}$ & $\checkmark$ \\
\hline Replay & $\checkmark$ & $\checkmark$ & $\checkmark$ & $\checkmark$ & $\checkmark$ & $\checkmark$ \\
\hline Parallel session & $\oiint^{1}$ & $\mathrm{~s}^{1}$ & $\mathrm{~s}^{1}$ & $\mathrm{~s}^{1}$ & $\xi^{1}$ & $\checkmark$ \\
\hline
\end{tabular}

${ }^{1}$ Untouched.

$\left.\left.\left.\left(r_{j}+r_{X}+U I D_{X}\right)\right) \bmod p\right) \bmod p\right) \bmod p$ because of facing CMDHP. Clearly, our scheme can resist this kind of attack.

4.10. User Impersonation Attack. To impersonate as a valid user, the adversary needs $R_{S}=T_{r_{S}} \quad\left(T_{A S I D_{j}} \quad\left(H_{0}\right.\right.$ $\left.\left.\left(r_{j}+r_{X}+U I D_{X}\right)\right) \bmod p\right) \bmod p$. To have $R_{S}$, he/she needs $U_{X}$ 's $R_{U}=T_{r_{U}}\left(T_{A S I D_{j}}\left(H_{0}\left(r_{j}+r_{X}+U I D_{X}\right)\right) \bmod p\right) \bmod p$. Furthermore, the adversary must resend the session key to $S_{j}$. Therefore, he/she not only finds $R_{U}$ but also knows $r_{U}$ to impersonate as a valid user. Clearly, our scheme can resist this kind of attack.
4.11. Server Impersonation Attack. To impersonate as a valid server, the adversary needs $R_{U}=T_{r_{U}}\left(T_{A S I D_{j}} \quad\left(H_{0}\right.\right.$ $\left.\left.\left(r_{j}+r_{X}+U I D_{X}\right)\right) \bmod p\right) \bmod p$. So, he/she also needs $A S I D_{j}$ to compute $R_{U}$. We see this is impossible because $S_{j}$ keeps $A S I D_{j}$ secret. Clearly, our scheme can resist this kind of attack.

4.12. Man-in-the-Middle Attack. In this kind of attack, the adversary can eavesdrop all messages exchanged between $U_{X}$ and $S_{j}$ and then edits the parameters in these packages. For example, the adversary can insert his/her own session key 
with randomly chosen $r_{U}$ and $r_{S}$. However, this is impossible because $U_{X}$ 's random value combined with $A S I D_{j}$. Therefore, the adversary needs to compute this key to achieve the goal, but this is impossible because this is $U_{X}$ 's secret key. Clearly, our scheme resists this attack.

4.13. Replay Attack. In this kind of attack, the adversary can eavesdrop all messages exchanged between $U_{X}$ and $S_{j}$. At any time, he/she can replay to cheat $U_{X}$ or $S_{j}$. We consider if the adversary resends $\left\{C I D, R^{\prime}, M_{U}, r_{X}\right\}$ to $S_{j}$, and this is the valid message. However, $S_{j}$ requests $U_{X}$ respond $\left\{M_{U S}\right\}$ for confirmation, and the adversary cannot compute $M_{U S}$. Furthermore, the adversary can resend $S_{j}$ 's $\left\{M_{S}, S^{\prime}\right\}$ to cheat $U_{X}$, but this is impossible because $U_{X}$ and $S_{j}$ use random values. So $\left\{M_{S}, S^{\prime}\right\}$ is only valid if $U_{X}$ rechooses random values. Clearly, our scheme can resist this kind of attack.

4.14. Parallel Session Attack. In this kind of attack, the adversary will use $\left\{M_{S}, S^{\prime}\right\}$ to create $\left\{C I D, R^{\prime}, M_{U}, r_{X}\right\}$, and cheat $S_{j}$. As aforementioned in replay and impersonation attacks, the adversary cannot achieve the goal because he/she needs key $U_{X}$ 's $T_{A S I D_{j}}\left(H_{0}\left(r_{j}+r_{X}+U I D_{X}\right)\right) \bmod p$ and $S_{j}$ 's $A S I D_{j}$. Clearly, our scheme resists this attack.

4.15. Efficiency Analysis. Now, this section presents the cost of our scheme compared with previous schemes. Before coming to detail comparison, we will unify some notations and the bit size of some cryptographic primitives. If the scheme does not mention, we assume as follows: the sizes of identity, password, biometrics, timestamp, and random values are 128 bits. The size of hash function's output and encryption scheme is 128 bits. The prime number in modular is 1024 bits $(\approx 309$ decimal digits) and 233 bits $(\approx 70$ decimal digits) in ECC. We let the notations denote the time to compute some cryptographic operations:

(i) $t_{\mathrm{H}}$ : time to compute hash function $(\approx 0.0004 \mathrm{~ms})$

(ii) $t_{\mathrm{PA}}$ : time to compute point addition $(\approx 0.36 \mathrm{~ms})$

(iii) $t_{\mathrm{PM}}$ : time to compute point multiplication $(\approx 12.4 \mathrm{~ms})$

(iv) $\mathrm{t}_{\mathrm{E} / \mathrm{D}}$ : time to encrypt/decrypt $(\approx 0.09 \mathrm{~ms})$

(v) $t_{\mathrm{T}}$ : time to compute Chebyshev polynomial $(\approx 127 \mathrm{~ms})$

All the amount of time is results we measure on the real Android device with the Bouncy Castle library. Next, we evaluate the computation quantity of previous schemes and ours in Table 8. In Li et al.'s scheme [11], registration needs $4 \times t_{\mathrm{H}}$ and authentication needs $15 \times t_{\mathrm{H}}$. In $\mathrm{Qu}$ and Tan's scheme [12], registration needs $4 \times t_{\mathrm{H}}, 2 \times t_{\mathrm{PM}}$ and authentication needs $11 \times t_{\mathrm{H}}, 5 \times t_{\mathrm{PM}}$ and $5 \times t_{\mathrm{PA}}$. In Amin and Biswas's scheme [13], registration needs $5 \times t_{\mathrm{H}}, 1 \times t_{\mathrm{E} / \mathrm{D}}$ and authentication needs $10 \times t_{\mathrm{H}}, 5 \times t_{\mathrm{PM}}, 2 \times t_{\mathrm{PA}}$ and $1 \times t_{\mathrm{E} / \mathrm{D}}$. In Jangirala et al.'s scheme [18], registration needs $5 \times t_{\mathrm{H}}, 1 \times t_{\mathrm{E} / \mathrm{D}}$ and authentication needs $10 \times t_{\mathrm{H}}, 5 \times t_{\mathrm{PM}}, 2 \times t_{\mathrm{PA}}$ and $1 \times t_{\mathrm{E} / \mathrm{D}}$. In Han et al.'s scheme [19], registration needs $5 \times t_{\mathrm{H}}, 1 \times t_{\mathrm{E} / \mathrm{D}}$ and authentication needs $10 \times t_{\mathrm{H}}, 5 \times t_{\mathrm{PM}}, 2 \times t_{\mathrm{PA}}$ and $1 \times t_{\mathrm{E} / \mathrm{D}}$.
TABLE 8: The comparison of computation cost.

\begin{tabular}{lc}
\hline Schemes & Authentication/Registration phases \\
\hline Li [11] & $\begin{array}{c}15 \times t_{\mathrm{H}} \\
4 \times t_{\mathrm{H}}\end{array}$ \\
\hline Qu [12] & $\begin{array}{c}11 \times t_{\mathrm{H}}, 5 \times t_{\mathrm{PM}}, 5 \times t_{\mathrm{PA}} \\
4 \times t_{\mathrm{H}}+2 \times t_{\mathrm{PM}}\end{array}$ \\
\hline Amin [13] & $10 \times t_{\mathrm{H}}, \begin{array}{c}5 \times t_{\mathrm{PM}}, 2 \times t_{\mathrm{PA}}, 1 \times t_{\mathrm{E} / \mathrm{D}} \\
5 \times t_{\mathrm{H}}, 1 \times t_{\mathrm{E} / \mathrm{D}}\end{array}$ \\
\hline Jangirala [18] & $\begin{array}{l}21 \times t_{\mathrm{H}} \\
6 \times t_{\mathrm{H}}\end{array}$ \\
\hline Han [19] & $11 \times t_{\mathrm{H}}, 2 \times t_{\mathrm{E} / \mathrm{D}}, 4 \times t_{\mathrm{T}}$ \\
$4 \times t_{\mathrm{H}}, 1 \times t_{\mathrm{E} / \mathrm{D}}$ \\
\hline Ours & $\begin{array}{l}11 \times t_{\mathrm{H}}, 6 \times t_{\mathrm{T}} \\
4 \times t_{\mathrm{H}}, 2 \times t_{\mathrm{T}}\end{array}$ \\
\hline
\end{tabular}

TABle 9: The comparison of storage authentication cost.

\begin{tabular}{lcc}
\hline Schemes & Storage cost (bits) & Authentication cost (bits) \\
\hline Li [11] & 640 & 896 \\
Qu [12] & 1618 & 3702 \\
Amin [13] & 2304 & 4818 \\
Jangirala [18] & 768 & 896 \\
Han [19] & 640 & 3072 \\
Ours & 722 & 2688 \\
\hline
\end{tabular}

In the proposed scheme, registration needs $2 \times t_{\mathrm{T}}, 4 \times t_{\mathrm{H}}$ and authentication needs $11 \times t_{\mathrm{H}}, 6 \times t_{\mathrm{T}}$.

Next, we evaluate the store and authentication costs of previous schemes and ours in Table 9. In Li et al.'s scheme [11], the user stores $\left\{C_{i}, D_{i}, E_{i}, h(),. h(y), b\right\}$ with 640 bits. And the authentication in this scheme needs $\left\{P_{i j}, C I D_{i}, M_{1}\right.$, $\left.M_{2}\right\},\left\{M_{3}, M_{4}\right\}$, and $\left\{M_{5}\right\}$ with 896 bits. In $\mathrm{Qu}$ and Tan's scheme [12], the user stores $\left\{A I D_{U}, B I D_{U}, b_{U}\right\}$ with 1618 bits. And the authentication in this scheme needs $\left\{C I D_{U}, D I D_{U}\right.$, $\left.E I D_{U}, R\right\},\left\{T, H_{S}\right\}$, and $\left\{H_{R S}\right\}$ with 3702 bits. In Amin and Biswas's scheme [13], the user needs $\left\{F_{i}, A_{i}, B_{i}, C I D_{i}, h(), H\right.$. (.) $\}$ with 2304 bits. And authentication in this scheme needs $\left\{C_{2}, C_{4}, C I D_{i}\right\},\left\{L_{i}, G_{1}, C I D_{i}^{\prime}\right\}$, and $\left\{Z_{i}\right\}$ with 4818 bits. In Jangirala et al.'s scheme [18], the user needs $\left\{C_{i}, D_{i}, E_{i}, h(y)\right.$, $h()$.$\} with 768$ bits. And authentication in this scheme needs $\left\{P_{i j}, C I D_{i}, M_{1}, M_{2}\right\},\left\{M_{3}, M_{4}\right\}$, and $\left\{M_{5}\right\}$ with 896 bits. In Han et al.'s scheme [19], the user needs $\{P, K, V, C I D, h()$.$\} with$ 640 bits. And authentication in this scheme needs $\{C I D, X$, $\left.V_{1}, T_{1}\right\},\left\{C I D^{\prime}, Y, T_{2}, V_{2}\right\}$, and $\left\{V_{3}, T_{3}\right\}$ with 3072 bits. In our proposed scheme, the user stores $\left\{s_{1_{X}}, s_{2_{X}}, \ldots, s_{m_{X}}, R P W_{X}, H_{0}\right.$ (.), $\left.r_{X}\right\}$, but we assume that the smart card stores only one $s_{i_{X}}$ for convenient comparison with other schemes in singleserver environment, so the cost is 722 bits. And authentication in our scheme needs $\left\{C I D, R^{\prime}, M_{U}, r_{X}\right\},\left\{M_{S}, S^{\prime}\right\}$, and $\left\{M_{U S}\right\}$ with 2688 bits.

\section{Conclusions}

This paper proposed a scheme using Chebyshev polynomial in multiserver environment. We survey and analysis current schemes to propose the solution overcoming the limitations in each approach. In the future, we will analyse many different approaches to apply with our scheme. Also, we design 
some new architectures which have average overall cost even if using high-cost computational operation. Finally, we try to minimize the size of message package exchanged between the user and server to enhance time-efficiency.

\section{Data Availability}

No data were used to support this study.

\section{Conflicts of Interest}

The authors declare that there are no conflicts of interest regarding the publication of this paper.

\section{Acknowledgments}

The authors would like to say a special thanks to the University of Science, Vietnam National University, Ho Chi Minh City, Vietnam. This research was funded by the University of Science, Vietnam National University, Ho Chi Minh City, Vietnam, under the grant number T2018-01.

\section{References}

[1] L. Lamport, "Password authentication with insecure communication," Communications of the ACM, vol. 24, no. 11, pp. $770-772,1981$.

[2] C. C. Lee, T. H. Lin, and R. X. Chang, "A secure dynamic ID based remote user authentication scheme for multi-server environment using smart cards," Expert Systems with Applications, vol. 38, no. 11, pp. 13863-13870, 2011.

[3] J. J. Shen, C. W. Lin, and M. S. Hwang, "A modified remote user authentication scheme using smart cards," IEEE Transactions on Consumer Electronics, vol. 49, no. 2, pp. 414-416, 2003.

[4] M. L. Das, A. Saxena, and V. P. Gulati, "A dynamic ID-based remote user authentication scheme," IEEE Transactions on Consumer Electronics, vol. 50, no. 2, pp. 629-631, 2004.

[5] I. E. Liao, C. C. Lee, and M. S. Hwang, "Security enhancement for a dynamic ID-based remote user authentication scheme," in Proceedings of the International Conference on Next Generation Web Services Practices, Seoul, Korea, 2005.

[6] Z.-Y. Wu, Y.-C. Lee, F. Lai, H.-C. Lee, and Y. Chung, "A secure authentication scheme for telecare medicine information systems," Journal of Medical Systems, vol. 36, no. 3, pp. 1529-1535, 2010.

[7] H. Debiao, C. Jianhua, and Z. Rui, "A more secure authentication scheme for tele-care medicine information systems," Journal of Medical Systems, vol. 36, no. 3, pp. 1589-1995, 2011.

[8] J. Wei, X. Hu, and W. Liu, "An improved authentication scheme for telecare medicine information systems," Journal of Medical Systems, vol. 36, no. 6, pp. 3597-3604, 2012.

[9] Z. Zhu, "An efficient authentication scheme for telecare medicine information systems," Journal of Medical Systems, vol. 36, no. 6, pp. 3833-3838, 2012.

[10] Q. Pu, J. Wang, and R. Zhao, "Strong authentication scheme for telecare medicine information systems," Journal of Medical Systems, vol. 36, no. 4, pp. 2609-2619, 2012.

[11] X. Li, J. Ma, W. Wang, Y. Xiong, and J. Zhang, "A novel smart card and dynamic ID based remote user authentication scheme for multi-server environments," Mathematical and Computer Modelling, vol. 58, no. 1-2, pp. 85-95, 2013.
[12] J. Qu and X.-L. Tan, “Two-factor user authentication with key agreement scheme based on elliptic curve cryptosystem," Journal of Electrical and Computer Engineering, vol. 2014, no. 4, pp. 1-6, 2014.

[13] R. Amin and G. P. Biswas, "A secure three-factor user authentication and key agreement protocol for TMIS with user anonymity," Journal of Medical Systems, vol. 39, no. 8, pp. 1-19, 2015.

[14] S. Qiu, G. Xu, H. Ahmad, and L. Wang, “A robust mutual authentication scheme based on elliptic curve cryptography for telecare medical information systems," IEEE Access, vol. 6, pp. 7452-7463, 2018.

[15] G. Xu, S. Qiu, H. Ahmad et al., "A multi-server two-factor authentication scheme with un-traceability using elliptic curve cryptography," Sensors, vol. 18, no. 7, p. 2394, 2018.

[16] S. Qiu, G. Xu, H. Ahmad, G. Xu, X. Qiu, and H. Xu, “An improved lightweight two-factor authentication and key agreement protocol with dynamic identity based on elliptic curve cryptography," KSII Transactions on Internet and Information Systems, vol. 13, no. 2, pp. 978-1002, 2019.

[17] X. Li, J. Niu, S. Kumari et al., "A novel chaotic maps-based user authentication and key agreement protocol for multiserver environments with provable security," Wireless Personal Communications, vol. 89, no. 2, pp. 569-597, 2016.

[18] S. Jangirala, S. Mukhopadhyay, and A. K. Das, "A multi-server environment with secure and efficient remote user authentication scheme based on dynamic ID using smart cards," Wireless Personal Communications, vol. 95, no. 3, pp. 27352767, 2017.

[19] L. Han, Q. Xie, W. Liu, and S. Wang, "A new efficient chaotic maps based three factor user authentication and key agreement scheme," Wireless Personal Communications, vol. 95, no. 3, pp. 3391-3406, 2017.

[20] A. Irshad, M. Sher, M. U. Ashraf et al., "An improved and secure chaotic-map based multi-server authentication protocol based on Lu et al. and Tsai and Lo's scheme," Wireless Personal Communications, vol. 95, no. 3, pp. 3185-3208, 2017.

[21] B. A. Alzahrani and A. Irshad, "A secure and efficient TMISbased authentication scheme improved against Zhang et al.'s scheme," Arabian Journal for Science and Engineering, vol. 43, no. 12, pp. 8239-8253, 2018.

[22] D. Wang and P. Wang, "Two birds with one stone: two-factor authentication with security beyond conventional bound," IEEE Transactions on Dependable and Secure Computing, vol. 15, no. 4, pp. 708-722, 2018.

[23] T.-T. Truong, M.-T. Tran, and A.-D. Duong, "Improved Chebyshev polynomials-based authentication scheme in client-server environment," Security and Communication Networks, vol. 2019, Article ID 4250743, 11 pages, 2019.

[24] L. Zhang, "Cryptanalysis of the public key encryption based on multiple chaotic systems," Chaos, Solitons \& Fractals, vol. 37, no. 3, pp. 669-674, 2008.

[25] P. Bergamo, P. D’Arco, A. De Santis, and L. Kocarev, "Security of public-key cryptosystems based on Chebyshev polynomials," IEEE Transactions on Circuits and Systems I: Regular Papers, vol. 52, no. 7, pp. 1382-1393, 2005.

[26] L. Kocarev and S. Lian, Chaos-Based Cryptography: Theory, Algorithms and Applications, Springer, Berlin, Germany, 2011.

[27] A. T. B. Jin, D. N. C. Ling, and A. Goh, "Biohashing: two factor authentication featuring fingerprint data and tokenised random number," Pattern Recognition, vol. 37, no. 11, pp. 2245-2255, 2004. 
[28] M. Burrows, M. Abadi, and R. Needham, "A logic of authentication," ACM Transactions on Computer Systems, vol. 8, no. 1, pp. 18-36, 1990.

[29] J.-L. Tsai, T.-C. Wu, and K.-Y. Tsai, "New dynamic ID authentication scheme using smart cards," International Journal of Communication Systems, vol. 23, no. 12, pp. 1449-1462, 2010.

[30] K.-H. Yeh, "A provably secure multi-server based authentication scheme," Wireless Personal Communications, vol. 79, no. 3, pp. 1621-1634, 2014. 